\title{
NON UNIVERSALITY OF FLUCTUATIONS OF OUTLIERS FOR HERMITIAN POLYNOMIALS IN A COMPLEX WIGNER MATRIX AND A SPIKED DIAGONAL MATRIX
}

\author{
MIREILLE CAPITAINE*
}

\begin{abstract}
We study the fluctuations associated to the a.s. convergence of the outliers established by Belinschi-Bercovici-Capitaine of an Hermitian polynomial in a complex Wigner matrix and a spiked deterministic real diagonal matrix. Thus, we extend the non universality phenomenon established by Capitaine-Donati Martin-Féral for additive deformations of complex Wigner matrices, to any Hermitian polynomial. The result is described using the operator-valued subordination functions of free probability theory.
\end{abstract}

Key words: Random matrices; Free probability; Outliers; Fluctuations; Nonuniversality; Linearization; Operator-valued subordination.

Mathematics Subject Classification 2000: 15A18, 15B52,60B20, 60F05, 46L54

\section{Introduction}

There is currently a quite precise knowledge of the asymptotic spectral properties (i.e. when the dimension of the matrix tends to infinity) of a number of "classical" random matrix models (Wigner matrices, Wishart matrices, invariant ensembles...). This understanding covers both the so-called global regime (asymptotic behavior of the spectral measure) and the local regime (asymptotic behavior of the extreme eigenvalues and eigenvectors, spacings...). We refer to the monographies [2, 5, 26, [28, 34, 37] for a thorough introduction to random matrix theory.

Practical problems (in the theory of statistical learning, signal detection etc.) naturally lead to wonder about the spectrum reaction of a given random matrix after a deterministic perturbation. For example, in the signal theory, the deterministic perturbation is seen as the signal, the perturbed matrix is perceived as a noise, and the question is to know whether the observation of the spectral properties of signal plus noise can give access to significant parameters on the signal. Theoretical results on these "deformed" random models may allow to establish statistical tests on these parameters. A typical illustration is the so-called BBP phenomenon (after Baik, Ben Arous, Péché [7]) which put forward outliers (eigenvalues that move away from the rest of the spectrum) and their Gaussian fluctuations for spiked covariance

\footnotetext{
* Institut de Mathématiques de Toulouse; UMR5219; Université de Toulouse; CNRS; UPS, 118 rte de Narbonne F-31062 Toulouse, FRANCE. E-mail: mireille.capitaine@math.univ-toulouse.fr
} 
matrices.

Péché 38 established Gaussian fluctuations for any outlier of a low rank additive deformation of a G.U.E. matrix. Fluctuations of outliers for additive finite rank deformations of non-Gaussian Wigner matrices have been studied in [20, 21, 27, 39, 40, It turns out that the limiting distribution depends on the localisation/delocalisation of the eigenvectors associated to the non-null eigenvalues of the perturbation. Note that in the G.U.E. case investigated by Péché [38, the eigenvectors of the perturbation are irrelevant for the fluctuations, due to the unitary invariance in Gaussian models. Let us illustrate this dependence on the eigenvectors of the perturbation in a very simple situation. Let $W_{N}=\left(W_{i j}\right)_{1 \leq i, j \leq N}$ be a $N \times N$ Hermitian Wigner matrix where $\left\{W_{i i}, \sqrt{2} \mathcal{R} W_{i j}, \sqrt{2} \mathcal{I} W_{i j}\right\}_{1<i<j}$ are independent identically distributed random variables with law $\mu, \mu$ is a symmetric distribution, with variance 1 , and satisfies a Poincaré inequality (see the Appendix). Note that when $\mu$ is Gaussian, $W_{N}$ is a G.U.E matrix. Consider two finite rank perturbations of rank 1, with one non-null eigenvalue $\theta>1$. The first one $A_{N}^{(1)}$ is a matrix with all entries equal to

$\theta / N$ (delocalized eigenvector associated to $\theta$ ). The second one $A_{N}^{(2)}$ is a diagonal matrix (localized eigenvector associated to $\theta$ ). The limiting spectral distribution of each matrix $M_{N}^{(i)}=\frac{W_{N}}{\sqrt{N}}+A_{N}^{(i)}(i=1,2)$ is the semi-circular distribution

$$
d \mu_{s c}(t)=\frac{1}{2 \pi} \sqrt{4-t^{2}} \mathbf{1}_{[-2 ; 2]}(t) d t
$$

Nevertheless the largest eigenvalue $\lambda_{1}$ of each matrix $M_{N}^{(i)}(i=1,2)$ separates from the bulk and converges towards $\rho_{\theta}:=\theta+\frac{1}{\theta}(>2)$. The fluctuations of $\lambda_{1}$ around $\rho_{\theta}$ are given as follows :

Proposition 1.1. 1. Delocalized case 27]: The largest eigenvalue $\lambda_{1}\left(M_{N}^{(1)}\right)$ have Gaussian fluctuations,

$$
\sqrt{N}\left(\lambda_{1}\left(M_{N}^{(1)}\right)-\rho_{\theta}\right) \stackrel{\mathcal{D}}{\longrightarrow} \mathcal{N}\left(0,1-1 / \theta^{2}\right) .
$$

2. Localized case [20]: The largest eigenvalue $\lambda_{1}\left(M_{N}^{(2)}\right)$ fluctuates as

$$
\sqrt{N}\left(1-\frac{1}{\theta^{2}}\right)\left(\lambda_{1}\left(M_{N}^{(2)}\right)-\rho_{\theta}\right) \stackrel{\mathcal{D}}{\longrightarrow} \mu \star \mathcal{N}\left(0, v_{\theta}\right) .
$$

where the variance $v_{\theta}$ of the Gaussian distribution depends on $\theta$ and the fourth moment of $\mu$.

Hence, for localized eigenvectors of the perturbation, the limiting distribution depends on the distribution of the entries of the Wigner matrix and thus, this uncovers a non universality phenomenon. This paper wants to extend such a non universality phenomenon for an additive deformation, to general polynomials in a Wigner matrix and a diagonal deterministic matrix. Free probability is a main tool to achieve this purpose. 
Free probability theory was introduced by Voiculescu around 1983 motivated by the isomorphism problem of von Neumann algebras of free groups. He developped a noncommutative probability theory, on a noncommutative probability space, in which a new notion of freeness plays the role of independence in classical probability. Around 1991, Voiculescu 43 threw a bridge connecting random matrix theory with free probability since he realized that the freeness property is also present for many classes of random matrices, in the asymptotic regime when the size of the matrices tends to infinity. Since then, several papers aimed at developing the contribution of free probability theory to the analysis of the spectral properties of deformed ensembles and polynomials in random matrices. In particular, the main principle of subordination in free probability is emphasized as a main tool in the understanding of the localization of the outliers and the corresponding eigenvectors of many matricial models. It was the purpose of [19] to put forward an unified understanding based on subordination in free probability for studying the spectral properties of full rank deformations of classical Hermitian matrix models. This investigation relies notably on [22, 16, 17, 10, 18. This universal understanding culminates in [12] dealing with noncommutative polynomials in random Hermitian matrices; this investigation is achieved by an even more general methodology based on a linearization procedure and operator-valued subordination properties.

The aim of this paper is to study the fluctuations associated to the a.s. convergence of the outliers described in [12] of an Hermitian polynomial in a Wigner matrix and a spiked deterministic Hermitian matrix (spiked means that the matrix has a fixed eigenvalue outside the support of its limiting spectral measure). Capitaine and Péché 23] established Gaussian fluctuations for any outlier of a full rank additive deformation of a G.U.E. matrix using scalar-valued free probability theory. We generalize this result to any polynomial in a G.U.E. matrix and a deterministic Hermitian matrix which has a spike with multiplicity one, using operator-valued free probability theory. Moreover, considering any Hermitian polynomial in a nonGaussian Wigner matrix and a deterministic real diagonal matrix which has a spike with multiplicity one, we establish that the limiting distribution of outliers is the classical convolution of a Gaussian distribution and the distribution of the entries of the Wigner matrix; thus, this extends the non universality phenomenon (1.2) established in 20] for additive deformations of Wigner matrices. The result is described in terms of operator-valued subordination functions related to a linearization of the noncommutative polynomial involved in the definition of our model. Therefore, we start by describing the necessary terminology and results concerning linearization procedure and free probability theory in Sections 2 and 3 . In Section 4, we present our matrix model and main results (Theorem 4.1 and Corollary 4.1). Section 5 gathers several preliminary results that will be used in Section 6 to prove Theorem 4.1. An Appendix recalls some basic facts on Poincaré inequalities and concentration phenomenon that are used in some proofs, as well as a basic development of the determinant of a perturbation of a matrix.

To begin with, we introduce some notations.

- $M_{p}(\mathbb{C})$ is the set of $p \times p$ matrices with complex entries, $M_{p}^{s a}(\mathbb{C})$ the subset of 
self-adjoint elements of $M_{p}(\mathbb{C})$ and $I_{p}$ the identity matrix. In the following, we shall consider two sets of matrices with $p=m$ ( $m$ fixed) and $p=N$ or $N-1$ with $N \longrightarrow \infty$.

- $\operatorname{Tr}_{p}$ denotes the trace and $\operatorname{tr}_{p}=\frac{1}{p} \operatorname{Tr}_{p}$ the normalized trace on $M_{p}(\mathbb{C})$.

- $\|$.$\| denotes the operator norm on M_{p}(\mathbb{C})$.

- $\operatorname{id}_{p}$ denotes the identity operator from $M_{p}(\mathbb{C})$ to $M_{p}(\mathbb{C})$.

- $\left(E_{i j}\right)_{i, j=1}^{N}\left(\right.$ resp. $\left.\left(E_{i j}\right)_{i, j=1}^{N-1}\right)$ denotes the canonical basis of $M_{N}(\mathbb{C})$ (resp. $\left.M_{N-1}(\mathbb{C})\right)$ whereas $\left(e_{p q}\right)_{p, q=1}^{m}$ denotes the canonical basis of $M_{m}(\mathbb{C})$.

For any integer number $k$, we will say that a random term in some $M_{p}(\mathbb{C})$, depending on $N$ and $w \in M_{m}(\mathbb{C})$ such that $\Im w$ is positive definite, is $O\left(\frac{1}{N^{k}}\right)$ if its operator norm is smaller than $\frac{Q\left(\left\|(\Im w)^{-1}\right\|\right)(\|w\|+1)^{d}}{N^{k}}$ for some deterministic polynomial $Q$ whose coefficients are nonnegative real numbers and some integer number $d$ (which may depend on $m$ ).

For a family of random terms $I_{i}, i \in\{1, \ldots, N\}^{2}$, we will set $I_{i}=O_{i}^{(u)}\left(\frac{1}{N^{k}}\right)$ if for each $i, I_{i}=O\left(\frac{1}{N^{k}}\right)$ and moreover one can find a bound of the norm of each $I_{i}$ as above involving a common polynomial $Q$ and a common $d$, that is not depending on $i$.

Throughout the paper, $K, C$ denote some positive constants that may depend on $m$ and vary from line to line.

\section{A Linearization trick}

A powerful tool to deal with noncommutative polynomials in random matrices or in operators is the so-called "linearization trick" that goes back to Haagerup and Thorbjørnsen [29, 30] in the context of operator algebras and random matrices (see [35]). We use the procedure introduced in [1, Proposition 3].

Given a polynomial $P \in \mathbb{C}\left\langle X_{1}, \ldots, X_{k}\right\rangle$, we call linearization of $P$ any $L_{P} \in$ $M_{m}(\mathbb{C}) \otimes \mathbb{C}\left\langle X_{1}, \ldots, X_{k}\right\rangle$ such that

$$
L_{P}:=\left(\begin{array}{ll}
0 & u \\
v & Q
\end{array}\right) \in M_{m}(\mathbb{C}) \otimes \mathbb{C}\left\langle X_{1}, \ldots, X_{k}\right\rangle
$$

where

1. $m \in \mathbb{N}$,

2. $Q \in M_{m-1}(\mathbb{C}) \otimes \mathbb{C}\left\langle X_{1}, \ldots, X_{k}\right\rangle$ is invertible,

3. $\mathrm{u}$ is a row vector and $\mathrm{v}$ is a column vector, both of size $m-1$ with entries in $\mathbb{C}\left\langle X_{1}, \ldots, X_{k}\right\rangle$, 
4. the polynomial entries in $Q, u$ and $v$ all have degree $\leq 1$,

5.

$$
P=-u Q^{-1} v .
$$

It is shown in [1] that, given a polynomial $P \in \mathbb{C}\left\langle X_{1}, \ldots, X_{k}\right\rangle$, there exist $m \in \mathbb{N}$ and a linearization $L_{P} \in M_{m}(\mathbb{C}) \otimes \mathbb{C}\left\langle X_{1}, \ldots, X_{k}\right\rangle$. The algebra of polynomials in noncommuting indeterminates $X_{1}, \ldots, X_{k}$ becomes a *-algebra by anti-linear extension of $\left(X_{i_{1}} X_{i_{2}} \cdots X_{i_{l}}\right)^{*}=X_{i_{l}} \cdots X_{i_{2}} X_{i_{1}},\left(i_{1}, \ldots, i_{l}\right) \in\{1, \ldots, k\}^{l}, l \in \mathbb{N} \backslash\{0\}$. It turns out that if $P$ is self-adjoint, $L_{P}$ can be chosen to be self-adjoint.

The well-known result about Schur complements (see 35, Chapter 10, Proposition 1]) yields then the following invertibility equivalence.

Lemma 2.1. Let $P=P^{*} \in \mathbb{C}\left\langle X_{1}, \ldots, X_{k}\right\rangle$ and let $L_{P} \in M_{m}(\mathbb{C}) \otimes \mathbb{C}\left\langle X_{1}, \ldots, X_{k}\right\rangle$ be a linearization of $P$ with the properties outlined above. Let $y=\left(y_{1}, \ldots, y_{k}\right)$ be a $k$-tuple of self-adjoint operators in a unital $\mathcal{C}^{*}$-algebra $\mathcal{A}$. Then, for any $z \in \mathbb{C}$, $z e_{11} \otimes 1_{\mathcal{A}}-L_{P}(y)$ is invertible if and only if $z 1_{\mathcal{A}}-P(y)$ is invertible and we have

$$
\left(z e_{11} \otimes 1_{\mathcal{A}}-L_{P}(y)\right)^{-1}=\left(\begin{array}{cc}
\left(z 1_{\mathcal{A}}-P(y)\right)^{-1} & \star \\
\star & \star
\end{array}\right) .
$$

Beyond the equivalence described above, we will use the following bound.

Lemma 2.2. [12] Let $z_{0} \in \mathbb{C}$ be such that $z_{0} 1_{\mathcal{A}}-P(y)$ is invertible. There exist two polynomials $T_{1}$ and $T_{2}$ in $k$ commutative indeterminates, with nonnegative coefficients, depending only on $L_{P}$, such that

$$
\begin{aligned}
\|\left(z_{0} e_{11} \otimes\right. & \left.1_{\mathcal{A}}-L_{P}(y)\right)^{-1} \| \\
& \leq T_{1}\left(\left\|y_{1}\right\|, \ldots,\left\|y_{k}\right\|\right)\left\|\left(z_{0} 1_{\mathcal{A}}-P(y)\right)^{-1}\right\|+T_{2}\left(\left\|y_{1}\right\|, \ldots,\left\|y_{k}\right\|\right) .
\end{aligned}
$$

Moreover, if the distance from $z_{0}$ to the spectrum of $P(y)$ is at least $\delta>0$, and for any $i \in\{1, \ldots, k\},\left\|y_{i}\right\| \leq C$, for some positive real numbers $\delta$ and $C$, then there exists a constant $\varepsilon>0$, depending only on $L_{P}, \delta, C$ such that the distance from 0 to the spectrum of $\left(z_{0} e_{11} \otimes 1_{\mathcal{A}}-L_{P}(y)\right)$ is at least $\varepsilon$.

\section{$3 \quad$ Free Probability Theory}

\subsection{Scalar-valued free probability theory}

For the reader's convenience, we recall the following basic definitions from free probability theory. For a thorough introduction to free probability theory, we refer to [4].

- A $\mathcal{C}^{*}$-probability space, resp. a $\mathcal{W}^{*}$-probability space, is a pair $(\mathcal{A}, \phi)$ consisting of a unital $\mathcal{C}^{*}$-algebra $\mathcal{A}$, resp. of a unital von Neumann algebra, and a state $\phi$ on $\mathcal{A}$ (i.e a linear map $\phi: \mathcal{A} \rightarrow \mathbb{C}$ such that $\phi\left(1_{\mathcal{A}}\right)=1$ and $\phi\left(a a^{*}\right) \geq 0$ 
for all $a \in \mathcal{A})$, resp. a normal state. $\phi$ is a trace if it satisfies $\phi(a b)=\phi(b a)$ for every $(a, b) \in \mathcal{A}^{2}$. A trace is said to be faithful if $\phi\left(a a^{*}\right)>0$ whenever $a \neq 0$. An element of $\mathcal{A}$ is called a noncommutative random variable.

- The $\star$-noncommutative distribution of a family $a=\left(a_{1}, \ldots, a_{k}\right)$ of noncommutative random variables in a $\mathcal{C}^{*}$-probability space $(\mathcal{A}, \phi)$ is defined as the linear functional $\mu_{a}: P \mapsto \phi\left(P\left(a, a^{*}\right)\right)$ defined on the set of polynomials in $2 k$ noncommutative indeterminates, where $\left(a, a^{*}\right)$ denotes the $2 k$-tuple $\left(a_{1}, \ldots, a_{k}, a_{1}^{*}, \ldots, a_{k}^{*}\right)$. For any self-adjoint element $a_{1}$ in $\mathcal{A}$, there exists a probability measure $\nu_{a_{1}}$ on $\mathbb{R}$ such that, for every polynomial $\mathrm{P}$, we have

$$
\mu_{a_{1}}(P)=\int P(t) \mathrm{d} \nu_{a_{1}}(t) .
$$

Then, we identify $\mu_{a_{1}}$ and $\nu_{a_{1}}$. If $\phi$ is faithful then the support of $\nu_{a_{1}}$ is the spectrum of $a_{1}$ and thus $\left\|a_{1}\right\|=\sup \left\{|z|, z \in \operatorname{support}\left(\nu_{\mathrm{a}_{1}}\right)\right\}$.

- A family of elements $\left(a_{i}\right)_{i \in I}$ in a $\mathcal{C}^{*}$-probability space $(\mathcal{A}, \phi)$ is free if for all $k \in \mathbb{N}$ and all polynomials $p_{1}, \ldots, p_{k}$ in two noncommutative indeterminates, one has

$$
\phi\left(p_{1}\left(a_{i_{1}}, a_{i_{1}}^{*}\right) \cdots p_{k}\left(a_{i_{k}}, a_{i_{k}}^{*}\right)\right)=0
$$

whenever $i_{1} \neq i_{2}, i_{2} \neq i_{3}, \ldots, i_{n-1} \neq i_{k}$ and $\phi\left(p_{l}\left(a_{i_{l}}, a_{i_{l}}^{*}\right)\right)=0$ for $l=1, \ldots, k$.

- A noncommutative random variable $x$ in a $\mathcal{C}^{*}$-probability space $(\mathcal{A}, \phi)$ is a standard semicircular variable if $x=x^{*}$ and for any $k \in \mathbb{N}$,

$$
\phi\left(x^{k}\right)=\int t^{k} d \mu_{s c}(t)
$$

where $d \mu_{s c}(t)=\frac{1}{2 \pi} \sqrt{4-t^{2}} \mathbf{1}_{[-2 ; 2]}(t) d t$ is the semicircular standard distribution.

- Let $k$ be a nonnull integer number. Denote by $\mathcal{P}$ the set of polynomials in $2 k$ noncommutative indeterminates. A sequence of families of variables $\left(a_{n}\right)_{n \geq 1}=\left(a_{1}(n), \ldots, a_{k}(n)\right)_{n \geq 1}$ in $\mathcal{C}^{*}$-probability spaces $\left(\mathcal{A}_{n}, \phi_{n}\right)$ converges, when $\mathrm{n}$ goes to infinity, respectively in distribution if the map $P \in \mathcal{P} \mapsto$ $\phi_{n}\left(P\left(a_{n}, a_{n}^{*}\right)\right)$ converges pointwise and strongly in distribution if moreover the map $P \in \mathcal{P} \mapsto\left\|P\left(a_{n}, a_{n}^{*}\right)\right\|$ converges pointwise.

Proposition 3.1. [25, Proposition 2.1] Let $x_{n}=\left(x_{1}(n), \ldots, x_{k}(n)\right)$ and $x=\left(x_{1}, \ldots, x_{k}\right)$ be $k$-tuples of self-adjoint variables in $\mathcal{C}^{*}$-probability spaces, $\left(\mathcal{A}_{n}, \phi_{n}\right)$ and $(\mathcal{A}, \phi)$, with faithful states. Then, the following assertions are equivalent.

- $x_{n}$ converges strongly in distribution to $x$,

- for any self-adjoint variable $h_{n}=P\left(x_{n}\right)$, where $P$ is a fixed polynomial, $\mu_{h_{n}}$ converges in weak-* topology to $\mu_{h}$ where $h=P(x)$. Moreover, the 
support of $\mu_{h_{n}}$ converges in Hausdorff distance to the support of $\mu_{h}$, that is: for any $\epsilon>0$, there exists $n_{0}$ such that for any $n \geq n_{0}$,

$$
\operatorname{supp}\left(\mu_{\mathrm{h}_{\mathrm{n}}}\right) \subset \operatorname{supp}\left(\mu_{\mathrm{h}}\right)+(-\epsilon,+\epsilon) .
$$

The symbol supp means the support of the measure.

Additive free convolution arises as natural analogue of classical convolution in the context of free probability theory. For two Borel probability measures $\mu$ and $\nu$ on the real line, one defines the free additive convolution $\mu \boxplus \nu$ as the distribution of $a+b$, where $a$ and $b$ are free self-adjoint random variables with distributions $\mu$ and $\nu$, respectively. We refer to [14, 33, 42, for the definitions and main properties of free convolutions. Let us briefly recall the fundamental analytic subordination properties [15, 44, 46] of this convolution. The analytic subordination phenomenon for free additive convolution was first noted by Voiculescu in 44 for free additive convolution of compactly supported probability measures. Biane [15] extended the result to free additive convolutions of arbitrary probability measures on $\mathbb{R}$. A new proof was given later, using a fixed point theorem for analytic self-maps of the upper half-plane [9. Note that such a subordination property allows to give a new definition of free additive convolution [24. Let us define the reciprocal CauchyStieltjes transform $F_{\mu}(z)=1 / g_{\mu}(z)$, which is an analytic self-map of the upper half-plane, where $g_{\mu}: z \in \mathbb{C} \backslash \mathbb{R} \mapsto \int \frac{1}{z-t} d \mu(t)$. Given Borel probability measures $\mu$ and $\nu$ on $\mathbb{R}$, there exist a unique pair of analytic functions $\omega_{1}, \omega_{2}: \mathbb{C}^{+} \rightarrow \mathbb{C}^{+}$such that

$$
F_{\mu}\left(\omega_{1}(z)\right)=F_{\nu}\left(\omega_{2}(z)\right)=F_{\mu \boxplus \nu}(z), \quad z \in \mathbb{C}^{+} .
$$

Moreover $\lim _{y \rightarrow+\infty} \omega_{j}(i y) / i y=1, j=1,2$ and

$$
\omega_{1}(z)+\omega_{2}(z)-z=F_{\mu \boxplus \nu}(z), \quad z \in \mathbb{C}^{+} .
$$

In particular (see [9]), for any $z \in \mathbb{C}^{+} \cup \mathbb{R}$ so that $\omega_{1}$ is analytic at $z, \omega_{1}(z)$ is the attracting fixed point of the self-map of $\mathbb{C}^{+}$defined by

$$
w \mapsto F_{\nu}\left(F_{\mu}(w)-w+z\right)-\left(F_{\mu}(w)-w\right) .
$$

A similar statement, with $\mu, \nu$ interchanged, holds for $\omega_{2}$. In particular, according to (3.2), we have for any $z \in \mathbb{C}^{+}$,

$$
g_{\mu \boxplus \nu}(z)=g_{\mu}\left(\omega_{1}(z)\right)=g_{\nu}\left(\omega_{2}(z)\right) .
$$

\subsection{Operator-valued free probability theory}

There exists an extension, operator-valued free probability theory, which still shares the basic properties of free probability but is much more powerful because of its wider domain of applicability. The concept of freeness with amalgamation and some of the relevant analytic transforms were introduced by Voiculescu in [45].

Definition 3.1. Let $\mathcal{M}$ be an algebra and $\mathcal{B} \subset \mathcal{M}$ be a unital subalgebra. A linear map $E: \mathcal{M} \rightarrow \mathcal{B}$ is a conditional expectation if $E(b)=b$ for all $b \in \mathcal{B}$ and 
$E\left(b_{1} a b_{2}\right)=b_{1} E(a) b_{2}$ for all $a \in \mathcal{M}$ and $b_{1}, b_{2}$ in $\mathcal{B}$. Then $(\mathcal{M}, E)$ is called a $\mathcal{B}$ valued probability space. If in addition $\mathcal{M}$ is a $\mathcal{C}^{*}$-algebra, $\mathcal{B}$ is a $\mathcal{C}^{*}$-subalgebra of $\mathcal{M}$ and $E$ is completely positive, then we have a $\mathcal{B}$-valued $\mathcal{C}^{*}$-probability space.

Example: Let $(\mathcal{A}, \phi)$ be a noncommutative probability space. Define

$$
\begin{gathered}
M_{2}(\mathcal{A}):=\left\{\left(\begin{array}{ll}
a & b \\
c & d
\end{array}\right), a, b, c, d \in \mathcal{A}\right\}, \quad E:=\mathrm{id}_{2} \otimes \phi \text { that is } \\
E\left[\left(\begin{array}{ll}
a & b \\
c & d
\end{array}\right)\right]=\left(\begin{array}{ll}
\phi(a) & \phi(b) \\
\phi(c) & \phi(d)
\end{array}\right) .
\end{gathered}
$$

$\left(M_{2}(\mathcal{A}), E\right)$ is an $M_{2}(\mathbb{C})$-valued probability space $\left(\mathbb{C} \approx \mathbb{C} 1_{\mathcal{A}}\right)$.

As in scalar-valued free probability, one defines [45] freeness with amalgamation over $\mathcal{B}$ via an algebraic relation similar to freeness, but involving $E$ and noncommutative polynomials with coefficients in $\mathcal{B}$.

Definition 3.2. Let $(\mathcal{M}, E: \mathcal{M} \rightarrow \mathcal{B})$ be an operator-valued probability space. The $\mathcal{B}$-valued distribution of a noncommutative random variable $a \in \mathcal{M}$ is given by all $\mathcal{B}$-valued moments $E\left(a b_{1} a b_{2} \cdots b_{n-1} a\right) \in \mathcal{B}, n \in \mathbb{N}, b_{0}, \ldots, b_{n-1} \in \mathcal{B}$.

Let $\left(A_{i}\right)_{i \in I}$ be a family of subalgebras with $\mathcal{B} \subset A_{i}$ for all $i \in I$. The subalgebras $\left(A_{i}\right)_{i \in I}$ are free with respect to $E$ or free with amalgamation over $\mathcal{B}$ if $E\left(a_{1} \cdots a_{n}\right)=$ 0 whenever $a_{j} \in A_{i_{j}}, i_{j} \in I, E\left(a_{j}\right)=0$, for all $j$ and $i_{1} \neq i_{2} \neq \cdots \neq i_{n}$.

Random variables in $\mathcal{M}$ or subsets of $\mathcal{M}$ are free with amalgamation over $\mathcal{B}$ if the algebras generated by $\mathcal{B}$ and the variables or the algebras generated by $\mathcal{B}$ and the subsets, respectively, are so.

A centred $\mathcal{B}$-valued semicircular random variable $s$ is uniquely determined by its variance $\eta: b \mapsto E(s b s)$; a characterization in terms of moments and cumulants via $\eta$ is provided by Speicher in [41.

The previous results of free subordination property in the scalar case are approached from an abstract coalgebra point of view by Voiculescu in [46] and this approach extends the results to the $\mathcal{B}$-valued case. In [13], Belinschi, Mai and Speicher develop an analytic theory. In order to describe operator-valued subordination property, we need some notation. If $\mathcal{A}$ is a unital $\mathcal{C}^{*}$-algebra and $b \in \mathcal{A}$, we denote by $\Re b=\left(b+b^{*}\right) / 2$ and $\Im b=\left(b-b^{*}\right) / 2 i$ the real and imaginary parts of $b$, so $b=\Re b+i \Im b$. For a self-adjoint operator $b \in \mathcal{A}$, we write $b \geq 0$ if the spectrum of $b$ is contained in $[0,+\infty)$ and $b>0$ if the spectrum of $b$ is contained in $(0,+\infty)$. The operator upper half-plane of $\mathcal{A}$ is the set $\mathbb{H}^{+}(\mathcal{A})=\{b \in \mathcal{A}: \Im b>0\}$.

Proposition 3.2. [46], [13] (see Theorem 5 p 259 [35]) Let $(\mathcal{M}, E: \mathcal{M} \rightarrow \mathcal{B})$ be an operator-valued $\mathcal{C}^{*}$-probability space. Let $x_{1}, x_{2} \in \mathcal{M}$ be self-adjoint variables which are free with amalgamation over $\mathcal{B}$.

There exist a unique pair of Fréchet analytic maps $\omega_{1}, \omega_{2}: \mathbb{H}^{+}(\mathcal{B}) \rightarrow \mathbb{H}^{+}(\mathcal{B})$ such that, for all $b \in \mathbb{H}^{+}(\mathcal{B})$,

$$
\Im \omega_{j}(b) \geq \Im b, j=1,2 \text {; }
$$




$$
\begin{aligned}
& \text { - } \quad \begin{aligned}
& E\left[\left(b-\left(x_{1}+x_{2}\right)\right)^{-1}\right]=E\left[\left(\omega_{1}(b)-x_{1}\right)^{-1}\right]=E\left[\left(\omega_{2}(b)-x_{2}\right)^{-1}\right], \\
&\left\{E\left[\left(\omega_{1}(b)-x_{1}\right)^{-1}\right]\right\}^{-1}+b=\left\{E\left[\left(\omega_{2}(b)-x_{2}\right)^{-1}\right]\right\}^{-1}+b \\
&=\omega_{1}(b)+\omega_{2}(b) .
\end{aligned}
\end{aligned}
$$

Moreover, if $b \in \mathbb{H}^{+}(\mathcal{B})$, then $\omega_{1}(b)$ is the unique fixed point of the map

$$
\begin{array}{r}
f_{b}: \mathbb{H}^{+}(\mathcal{B}) \rightarrow \mathbb{H}^{+}(\mathcal{B}), \quad f_{b}(w)=h_{x_{2}}\left(h_{x_{1}}(w)+b\right)+b \\
\text { where } h_{x_{i}}(b)=E\left[\left(b-x_{i}\right)^{-1}\right]^{-1}-b \\
\text { and } \omega_{1}(b)=\lim _{k \rightarrow+\infty} f_{b}^{\circ k}(w), \text { for any } w \in \mathbb{H}^{+}(\mathcal{B}) .
\end{array}
$$

The following result from [36 explains why the particular case $\mathcal{B}=M_{m}(\mathbb{C})$, $\mathcal{M}=M_{m}(\mathcal{A}), E=\mathrm{id}_{m} \otimes \phi$, where $(\mathcal{A}, \phi)$ is a noncommutative probability space, is relevant in our work using linearizations of polynomials.

Proposition 3.3. Let $(\mathcal{A}, \phi)$ be a $\mathcal{C}^{*}$-probability space, let $m$ be a positive integer, and let $x_{1}, \ldots, x_{n} \in \mathcal{A}$ be freely independent. Then the map $\operatorname{id}_{m} \otimes \phi: M_{m}(\mathcal{A}) \rightarrow$ $M_{m}(\mathbb{C})$ is a unit preserving conditional expectation, and $\alpha_{1} \otimes x_{1}, \ldots, \alpha_{n} \otimes x_{n}$ are free over $M_{m}(\mathbb{C})$ for any $\alpha_{i} \in M_{m}(\mathbb{C})$.

Now, if $x$ is a standard scalar-valued semicircular centred noncommutative random variable which is free from a self-adjoint variable $a$ in some $\mathcal{W}^{*}$-probability space $(\mathcal{A}, \phi)$, then, for any Hermitian matrices $\alpha, \beta$ in $M_{m}(\mathbb{C}), \alpha \otimes x$ is a $M_{m}(\mathbb{C})$ valued semicircular of variance $\eta: b \mapsto \alpha b \alpha$ which is free over $M_{m}(\mathbb{C})$ from $\beta \otimes a$ and the subordination function has a more explicit form (see [35, Chapter 9] and the end of the proof of Theorem 8.3 in $[3])$ : for $b \in \mathbb{H}^{+}\left(M_{m}(\mathbb{C})\right)$,

$$
\left(\mathrm{id}_{m} \otimes \phi\right)\left[\left(b \otimes 1_{\mathcal{A}}-\alpha \otimes x-\beta \otimes a\right)^{-1}\right]=\left(\operatorname{id}_{m} \otimes \phi\right)\left[\left(\omega_{m}(b) \otimes 1_{\mathcal{A}}-\beta \otimes a\right)^{-1}\right],
$$

where

$$
\omega_{m}(b)=b-\alpha\left(\operatorname{id}_{m} \otimes \phi\right)\left[\left(b \otimes 1_{\mathcal{A}}-\alpha \otimes x-\beta \otimes a\right)^{-1}\right] \alpha .
$$

Denote by $\mathcal{N}$ the unital von Neumann algebra generated by $M_{m}(\mathbb{C})$ and $\beta \otimes a$ and by $E_{\mathcal{N}}$ the unique trace preserving conditional expectation of $M_{m}(\mathcal{A})$ onto $\mathcal{N}$. Actually the following strengthened result [46, Theorem 3.8] holds:

$$
E_{\mathcal{N}}\left[\left(b \otimes 1_{\mathcal{A}}-\alpha \otimes x-\beta \otimes a\right)^{-1}\right]=\left(\omega_{m}(b) \otimes 1_{\mathcal{A}}-\beta \otimes a\right)^{-1} .
$$




\section{Assumptions and main results}

\section{Assumptions on the Wigner matrix.}

We consider a $N \times N$ Hermitian Wigner matrix $W_{N}=\left(W_{i j}\right)_{1 \leq i, j \leq N}$ such that the random variables $\left\{W_{i i}, \sqrt{2} \mathcal{R} W_{i j}, \sqrt{2} \mathcal{I} W_{i j}\right\}_{1 \leq i<j}$ are independent identically distributed with law $\mu, \mu$ is a centered distribution, with variance 1 , and satisfies a Poincaré inequality (see the Appendix). We set

$$
W_{N}=\left(\begin{array}{cc}
W_{11} & Y^{*} \\
Y & W_{N-1}
\end{array}\right)
$$

where $Y^{*}=\left(W_{12}, \cdots, W_{1 N}\right)$ and $W_{N-1} \in M_{N-1}(\mathbb{C})$.

\section{Assumptions on the deterministic matrix.}

We consider a deterministic real diagonal matrix $A_{N}$ :

$$
A_{N}=\operatorname{diag}\left(\theta, A_{N-1}\right)
$$

where $\theta \in \mathbb{R}$ is independent of $N$ and $A_{N-1}$ is a $N-1 \times N-1$ deterministic diagonal matrix such that for any $i=1, \ldots, N-1,\left(A_{N-1}\right)_{i i}=d_{i}(N)$. We assume that $A_{N-1} \in\left(M_{N-1}(\mathbb{C}), \frac{1}{N-1} \operatorname{Tr}\right)$ converges strongly in distribution towards a noncommutative self-adjoint random variable $a$ in some $\mathcal{W}^{*}$-probability space $(\mathcal{A}, \phi)$, with $\phi$ faithful (see Section 3.1 for the definition of strong convergence). Note that this implies that

$$
\sup _{N}\left\|A_{N-1}\right\|<+\infty
$$

and, by Proposition 3.1. that, for all large $N$, all the eigenvalues of $A_{N-1}$ are in any small neighborhood of the spectrum of $a$. We assume that $\theta$ is such that $\theta \notin \operatorname{supp}\left(\mu_{a}\right)=\operatorname{spect}(a)$. Note that the previous assumptions yield that $A_{N} \in$ $\left(M_{N}(\mathbb{C}), \frac{1}{N} \operatorname{Tr}\right)$ converges in distribution towards the noncommutative random variable $a$ and that, for $N$ large enough, $\theta$ is an eigenvalue of multiplicity 1 of $A_{N}$.

\section{Matrix model.}

Fix a self-adjoint polynomial $P \in \mathbb{C}<X_{1}, X_{2}>$. The matrix model we are interested in is

$$
M_{N}=P\left(\frac{W_{N}}{\sqrt{N}}, A_{N}\right) .
$$

Denote by $\lambda_{i}\left(M_{N}\right), i=1, \ldots, N$, its eigenvalues and by

$$
\mu_{M_{N}}=\frac{1}{N} \sum_{i=1}^{N} \lambda_{i}\left(M_{N}\right)
$$

its empirical spectral measure. According to (2.10) in [11] and [2, Theorem 5.4.5], we have

$$
\lim _{N \rightarrow \infty} \mu_{M_{N}}=\mu_{P(x, a)}
$$

almost surely in the weak* topology, where $x$ is a standard semicircular noncommutative random variable in $(\mathcal{A}, \phi)$ (i.e $\left.d \mu_{x}=\frac{1}{2 \pi} \sqrt{4-x^{2}} \mathbf{1}_{[-2,2]}(x)\right), a$ and $x$ are 
freely independent, and $\mu_{P(x, a)}$ denotes the distribution of $P(x, a)$.

The set of outliers of $M_{N}$ is calculated in 12 from the spike $\theta$ of $A_{N}$ using linearization and Voiculescu's matrix subordination function [46] as follows. Choose a linearization $L_{P}$ of $P$ where $L_{P}=\gamma \otimes 1+\alpha \otimes X_{1}+\beta \otimes X_{2}, \alpha, \beta, \gamma$ are self-adjoint matrices in $M_{m}(\mathbb{C})$, and let $\omega_{m}$ be the subordination function associated to the semicircular operator-valued random variable $\alpha \otimes x$ with respect to $\beta \otimes a$, as defined by (3.5). According to Lemma2.1] $\omega_{m}$ extends as an analytic map $z \mapsto \omega_{m}\left(z e_{11}-\gamma\right)$ to $\mathbb{C} \backslash \operatorname{supp}\left(\mu_{P(x, a)}\right)$. For any $\rho \notin \operatorname{supp}\left(\mu_{P(x, a)}\right)$, define $m(\rho)$ as the multiplicity of $\rho$ as a zero of $\operatorname{det}\left(\omega_{m}\left(\rho e_{11}-\gamma\right)-\theta \beta\right)$. [12 establishes the following.

Proposition 4.1. [12] There exists $\delta_{0}>0$ such that, for any $0<\delta \leq \delta_{0}$, a.s for large $N$, there are exactly $m(\rho)$ eigenvalues of $P\left(\frac{W_{N}}{\sqrt{N}}, A_{N}\right)$ in $] \rho-\delta ; \rho+\delta[$, counting multiplicity.

\section{Assumptions on $\rho$.}

In this paper, we assume that there exists some real number

$$
\rho \notin \operatorname{supp}\left(\mu_{P(x, a)}\right)=\operatorname{spect}(P(x, a))
$$

such that $\rho$ is a zero with multiplicity one of

$$
\operatorname{det}\left(\omega_{m}\left(\rho e_{11}-\gamma\right)-\theta \beta\right)=0,
$$

that is such that

$$
m(\rho)=1
$$

Assumptions on $\epsilon$.

Throughout the paper $\epsilon>0$ is fixed such that

$$
d(\rho, \operatorname{spect}(P(x, a)))>\epsilon
$$

and

$$
\left.\operatorname{det}\left(\omega_{m}\left(y e_{11}-\gamma\right)-\theta \beta\right) \neq 0, \text { for any } y \in\right] \rho-\epsilon ; \rho+\epsilon[\backslash\{\rho\} \text {. }
$$

\section{Main result.}

We first introduce events and objects needed to state our main result.

By strong asymptotic freeness of [12] and Proposition 3.1] almost surely for all large $N, \operatorname{spect}\left(P\left(\frac{W_{N-1}}{\sqrt{N}}, A_{N-1}\right)\right) \subset\{y \in \mathbb{R} ; d(y, \operatorname{spect}(P(x, a)) \leq \epsilon / 2\}$. Thus,

$$
\text { almost surely for all large } N, d\left(\rho, \operatorname{spect}\left(P\left(\frac{W_{N-1}}{\sqrt{N}}, A_{N-1}\right)\right)\right)>\epsilon / 2 \text {. }
$$

Define the event

$$
\tilde{\Omega}_{N-1}=\left\{d\left(\rho, \operatorname{spect}\left(P\left(\frac{W_{N-1}}{\sqrt{N}}, A_{N-1}\right)\right)\right)>\epsilon / 2 ;\left\|\frac{W_{N-1}}{\sqrt{N}}\right\| \leq 3\right\},
$$


Note that according to Lemma 2.2, there exists $C_{\epsilon}>0$ such that

$$
d\left(0, \operatorname{spect}\left(\left(\rho e_{11}-\gamma\right) \otimes 1_{\mathcal{A}}-\alpha \otimes x-\beta \otimes a\right)\right)>C_{\epsilon}
$$

and on $\tilde{\Omega}_{N-1}$

$$
d\left(0, \text { spect }\left(\left(\rho e_{11}-\gamma\right) \otimes I_{N-1}-\alpha \otimes \frac{W_{N-1}}{\sqrt{N}}-\beta \otimes A_{N-1}\right)\right)>C_{\epsilon} .
$$

Let $\delta_{0}$ be as defined in Proposition 4.1 Set

$$
\tau=\min \left(\delta_{0}, \epsilon / 4, C_{\epsilon} / 4\right) .
$$

Define the event

$$
\Omega_{N}=\tilde{\Omega}_{N-1} \cap\left\{\operatorname{card}\left(\operatorname{spect}\left(M_{N}\right) \cap\right] \rho-\tau ; \rho+\tau[)=1\right\} .
$$

It readily follows from Proposition 4.1 4.5) and Bai-Yin's theorem (see Theorem 5.1 in [5]) that

$$
\lim _{N \rightarrow+\infty} \mathbf{1}_{\Omega_{N}}=1 \text {, a.s. }
$$

and then

$$
\mathbb{P}\left(\Omega_{N}\right) \rightarrow_{N \rightarrow+\infty} 1 .
$$

Now, define

$$
\lambda(N, \rho)=\left\{\begin{array}{l}
\left.\rho \text { if } \operatorname{spect}\left(M_{N}\right) \cap\right] \rho-\tau ; \rho+\tau[=\emptyset \\
\max \left\{\operatorname{spect}\left(M_{N}\right) \cap\right] \rho-\tau ; \rho+\tau[\} \text { else. }
\end{array}\right.
$$

On $\Omega_{N}, \lambda(N, \rho)$ is the unique eigenvalue of $M_{N}$ which is located in $] \rho-\tau ; \rho+\tau[$. In this paper, we study the fluctuations of $\lambda(N, \rho)$. Note that Proposition 4.1 readily implies that

$$
\lambda(N, \rho) \rightarrow_{N \rightarrow+\infty} \rho \text { a.s.. }
$$

Let $a_{N-1}$ be a self-adjoint noncommutative random variable in $(\mathcal{A}, \phi)$ whose distribution is $\mu_{A_{N-1}}$ (meaning that $\forall k \in \mathbb{N}, \frac{1}{N-1} \operatorname{Tr}\left(A_{N-1}^{k}\right)=\phi\left(\left(a_{N-1}^{k}\right)\right)$ and which is free with the semicircular variable $x$. Since $A_{N-1}$ (and thus $a_{N-1}$ ) converges strongly to $a$, we have, for all large $N$,

$$
\left.\operatorname{spect}\left(P\left(x, a_{N-1}\right)\right) \subset \operatorname{spect}(P(x, a))+\right]-\epsilon / 4, \epsilon / 4[,
$$

and thus, using (4.4), for any $z \in B(\rho, \tau):=\{z \in \mathbb{C},|z-\rho|<\tau\}$,

$$
\left\|\left(z 1_{\mathcal{A}}-P\left(x, a_{N-1}\right)\right)^{-1}\right\| \leq 2 / \epsilon .
$$

Define for any $\kappa \in \mathbb{H}^{+}\left(M_{m}(\mathbb{C})\right)$

$$
\omega_{m}^{(N)}(\kappa)=\kappa-\alpha\left(\mathrm{id}_{m} \otimes \phi\right)\left[\left(\kappa \otimes 1_{\mathcal{A}}-\alpha \otimes x-\beta \otimes a_{N-1}\right)^{-1}\right] \alpha .
$$

$\omega_{m}^{(N)}$ is the subordination function associated to the semicircular operator-valued random variable $\alpha \otimes x$ with respect to $\beta \otimes a_{N-1}$. According to Lemma 2.1. $\omega_{m}^{(N)}$ 
extends as an analytic map $z \mapsto \omega_{m}^{(N)}\left(z e_{11}-\gamma\right)$ to $\mathbb{C} \backslash \operatorname{supp}\left(\mu_{P\left(x, a_{N-1}\right)}\right)$. Using (4.13)

and Lemma2.2. it is straightforward to see that $\left(z \mapsto \operatorname{det}\left(\omega_{m}^{(N)}\left(z e_{11}-\gamma\right)-\theta \beta\right)\right)_{N \geq 1}$ is a bounded sequence in the set of analytic functions endowed with the uniform convergence on compact subsets of $B(\rho, \tau)$; therefore, using moreover (5.16) and Vitali's theorem, by Hurwitz's theorem, (4.2) yields that for any $0<\tau^{\prime}<\tau$, for all large $N$, there exists one and only one $\rho_{N}$ in $B\left(\rho, \tau^{\prime}\right)$, such that

$$
\operatorname{det}\left(\omega_{m}^{(N)}\left(\rho_{N} e_{11}-\gamma\right)-\theta \beta\right)=0
$$

and we have

$$
\rho_{N} \rightarrow_{N \rightarrow+\infty} \rho .
$$

Moreover, necessarily $\rho_{N}$ is real since (4.15) implies that $\operatorname{det}\left(\omega_{m}^{(N)}\left(\overline{\rho_{N}} e_{11}-\gamma\right)-\theta \beta\right)=$ 0 .

Here is our main result. (For a matrix $X, \operatorname{com}(X)$ denotes the comatrix of $X$.)

Theorem 4.1. Define

$$
\begin{gathered}
\mathbf{C}_{m}={ }^{t} \operatorname{com}\left(\omega_{m}\left(\rho e_{11}-\gamma\right)-\beta \theta\right) \\
R_{\infty}\left(\rho e_{11}-\gamma\right)=\left(\left(\rho e_{11}-\gamma\right) \otimes 1_{\mathcal{A}}-\alpha \otimes x-\beta \otimes a\right)^{-1} \\
C_{\rho}^{(1)}=\operatorname{Tr}_{m}\left(\mathbf{C}_{m}\left[e_{11}+\alpha\left(\operatorname{id}_{m} \otimes \phi\right)\left(R_{\infty}\left(\rho e_{11}-\gamma\right)\left(e_{11} \otimes 1_{\mathcal{A}}\right) R_{\infty}\left(\rho e_{11}-\gamma\right)\right) \alpha\right]\right), \\
C_{\rho}^{(2)}=\operatorname{Tr}_{m}\left[\mathbf{C}_{m} \alpha\right] \\
v_{\rho}=\left(\mathbb{E}\left(\left|W_{21}\right|^{4}\right)-2\right) \int\left[\operatorname{Tr}_{m}\left(\alpha \mathbf{C}_{m} \alpha\left(\omega_{m}\left(\rho e_{11}-\gamma\right)-t \beta\right)^{-1}\right)\right]^{2} d \mu_{a}(t) \\
+\phi\left(\left[\left(\operatorname{Tr}_{m} \otimes \operatorname{id}_{\mathcal{A}}\right)\left\{R_{\infty}\left(\rho e_{11}-\gamma\right)\left(\alpha \mathbf{C}_{m} \alpha\right) \otimes 1_{\mathcal{A}}\right\}\right]^{2}\right)
\end{gathered}
$$

with $\omega_{m}$ defined by (3.5).

$C_{\rho}^{(1)} \sqrt{N}\left(\lambda(N, \rho)-\rho_{N}\right)$ converges in distribution to the classical convolution of the distribution of $C_{\rho}^{(2)} W_{11}$ and a Gaussian distribution with mean 0 and variance $v_{\rho}$.

Using the unitarily invariance of the distribution of a G.U.E. matrix, we can readily deduce the following result.

Corollary 4.1. Assume that $W_{N}$ is a G.U.E. matrix. Let $A_{N}$ be a deterministic Hermitian matrix such that its spectral measure $\mu_{A_{N}}$ weakly converges towards a compactly supported measure $\mu_{a}, \theta \notin \operatorname{supp}\left(\mu_{a}\right)$ is a spiked eigenvalue of $A_{N}$ with multiplicity one whereas the other eigenvalues of $A_{N}$ converge uniformly to the compact support of $\mu_{a}$. Then, under the assumptions (4.2) and (4.3), $C_{\rho}^{(1)} \sqrt{N}\left(\lambda(N, \rho)-\rho_{N}\right)$ converges in distribution to a Gaussian distribution with mean 0 and variance

$$
\tilde{v}_{\rho}=\left(C_{\rho}^{(2)}\right)^{2}+\phi\left(\left[\left(\operatorname{Tr}_{m} \otimes \mathrm{id}_{\mathcal{A}}\right)\left\{R_{\infty}\left(\rho e_{11}-\gamma\right)\left(\alpha \mathbf{C}_{m} \alpha\right) \otimes 1_{\mathcal{A}}\right\}\right]^{2}\right),
$$

where $\lambda(N, \rho), \rho_{N}, C_{\rho}^{(1)}, C_{\rho}^{(2)}, \mathbf{C}_{m}$ and $R_{\infty}\left(\rho e_{11}-\gamma\right)$ are defined by (4.11), (4.15), (4.19), (4.20), (4.17) and (4.18) respectively. 


\section{Example}

As an illustration, consider the random matrix

$$
M_{N}=A_{N} \frac{W_{N}}{\sqrt{N}}+\frac{W_{N}}{\sqrt{N}} A_{N}+\frac{W_{N}^{2}}{N},
$$

where $W_{N}$ is a Wigner matrix of size $N$ such that $d \mu(x)=\frac{1}{2 \sqrt{3}} \mathbf{1}_{[-\sqrt{3} ; \sqrt{3}]}(x) d x$ and

$$
A_{N}=\operatorname{Diag}(\theta, 0, \ldots, 0), \quad \theta \in \mathbb{R} \backslash\{0\} .
$$

According to [8, (4.6.6)], $\mu$ satisfies a Poincaré inequality. In this case, $A_{N}$ has rank one, and thus $a=0$. It follows that the limit spectral measure $\Pi$ of $M_{N}$ is the same as the limit spectral measure of $W_{N}^{2} / N$. Thus, $\Pi$ is the Marchenko-Pastur distribution with parameter 1:

$$
d \Pi(t)=\frac{\sqrt{(4-t) t}}{2 \pi t} 1_{(0,4)}(t) d t .
$$

The polynomial $P$ is $P\left(X_{1}, X_{2}\right)=X_{2} X_{1}+X_{1} X_{2}+X_{1}^{2}, a=0$ and $x$ is the standard semi-circular distribution. An economical linearization of $P$ is provided by $L=$ $\gamma \otimes 1+\alpha \otimes X_{1}+\beta \otimes X_{2}$, where

$$
\gamma=\left[\begin{array}{ccc}
0 & 0 & 0 \\
0 & 0 & -1 \\
0 & -1 & 0
\end{array}\right], \quad \alpha=\left[\begin{array}{ccc}
0 & 1 & \frac{1}{2} \\
1 & 0 & 0 \\
\frac{1}{2} & 0 & 0
\end{array}\right], \quad \beta=\left[\begin{array}{ccc}
0 & 0 & 1 \\
0 & 0 & 0 \\
1 & 0 & 0
\end{array}\right] .
$$

Thus, here $m=3$. Denote by

$$
G_{\Pi}(z)=\int_{0}^{4} \frac{1}{z-t} d \Pi(t)=\frac{z-\sqrt{z^{2}-4 z}}{2 z}, \quad z \in \mathbb{C} \backslash[0,4],
$$

the Cauchy transform of the measure $\Pi$. This function satisfies the quadratic equation $z G_{\Pi}(z)^{2}-z G_{\Pi}(z)+1=0$. Suppose now that $t \notin[0,4]$. Denoting by $E=\mathrm{id}_{3} \otimes \phi: \mathrm{M}_{3}(\mathcal{A}) \rightarrow \mathrm{M}_{3}(\mathbb{C})$ the usual expectation, since $a=0$, the function $\omega_{3}$ is computed as follows:

$$
\omega_{3}\left(t e_{11}-\gamma\right)=E\left(\left(t e_{11}-\gamma-\alpha \otimes x\right)^{-1}\right)^{-1}, \quad t \in \mathbb{R} \backslash[0,4] .
$$

The inverse of $t e_{11}-\gamma-\alpha \otimes x$ is then calculated explicitly and application of the expected value to its entries yields

$$
\omega_{3}\left(t e_{11}-\gamma\right)=\left[\begin{array}{ccc}
\frac{1}{G_{\Pi}(t)} & 0 & 0 \\
0 & \frac{1}{t G_{\Pi}(t)}-1 & \frac{1}{2 t G_{\Pi}(t)}+\frac{1}{2} \\
0 & \frac{1}{2 t G_{\Pi}(t)}+\frac{1}{2} & \frac{1}{4 t G_{\Pi}(t)}-\frac{1}{4}
\end{array}\right] .
$$

The equation $\operatorname{det}\left[\beta \theta-\omega\left(t e_{11}-\gamma\right)\right]=0$ is easily seen to reduce to

$$
\theta^{2} G_{\Pi}(t)^{2}-\left(1-G_{\Pi}(t)\right)=0 .
$$


Thus, the matrix $M_{N}$ exhibits one (negative) outlier when $0<|\theta| \leq \sqrt{2}$

$$
\rho_{\theta}^{-}=\frac{2 \theta^{4}}{-\left(3 \theta^{2}+1\right)-\sqrt{4 \theta^{2}+1}\left(\theta^{2}+1\right)},
$$

and two outliers (one negative and one $>4$ ) when $|\theta|>\sqrt{2}$ :

$$
\rho_{\theta}^{ \pm}=\frac{2 \theta^{4}}{-\left(3 \theta^{2}+1\right) \pm \sqrt{4 \theta^{2}+1}\left(\theta^{2}+1\right)}
$$

note that

$$
g_{\rho_{\theta}^{ \pm}}=G_{\Pi}\left(\rho_{\theta}^{ \pm}\right)=\frac{1}{2}+\frac{-\left(\theta^{2}+1\right) \pm \sqrt{4 \theta^{2}+1}}{2 \theta^{2}} .
$$

Let $\rho$ be any of the two solutions $\rho_{\theta}^{+}$and $\rho_{\theta}^{-}$and set

$$
g_{\rho}=G_{\Pi}(\rho) .
$$

Note that since here $a_{N-1}=a=0$, we have $\rho_{N}=\rho$. After computations

$$
\begin{gathered}
\mathbf{C}_{3}=\left(\begin{array}{ccc}
g_{\rho}-1 & \frac{\theta}{2}\left(g_{\rho}-2\right) & -g_{\rho} \theta \\
\frac{\theta}{2}\left(g_{\rho}-2\right) & -\left(\frac{1}{4}+\theta^{2}\right) & -\frac{1}{g_{\rho}}+\frac{1}{2} \\
-g_{\rho} \theta & -\frac{1}{g_{\rho}}+\frac{1}{2} & -1
\end{array}\right), \\
R_{\infty}\left(\rho e_{11}-\gamma\right)=\left(\begin{array}{ccc}
\left(\rho-x^{2}\right)^{-1} & \frac{1}{2} x\left(\rho-x^{2}\right)^{-1} & x\left(\rho-x^{2}\right)^{-1} \\
\frac{1}{2} x\left(\rho-x^{2}\right)^{-1} & \frac{1}{4} x^{2}\left(\rho-x^{2}\right)^{-1} & 1+\frac{1}{2} x^{2}\left(\rho-x^{2}\right)^{-1} \\
x\left(\rho-x^{2}\right)^{-1} & 1+\frac{1}{2} x^{2}\left(\rho-x^{2}\right)^{-1} & x^{2}\left(\rho-x^{2}\right)^{-1}
\end{array}\right),
\end{gathered}
$$

and then

$$
\begin{gathered}
C_{\rho}^{(2)}=-2 \theta \\
C_{\rho}^{(1)}=-\theta^{2} g_{\rho}^{2}\left(1+\int \frac{y}{(\rho-y)^{2}} d \Pi(y)\right)-\frac{1}{g_{\rho}^{2}} \int \frac{1}{(\rho-y)^{2}} d \Pi(y)<0
\end{gathered}
$$

and

$$
\begin{aligned}
v_{\rho}= & -\frac{3}{5}\left(\theta^{2} g_{\rho}+2\right)^{2} \\
+ & \theta^{4} g_{\rho}^{4} \int \frac{y^{2}}{(\rho-y)^{2}} d \mu_{\Pi}(y)+2 \theta^{2}\left(2+\theta^{2} g_{\rho}^{2}+g_{\rho}\right) \int \frac{y}{(\rho-y)^{2}} d \mu_{\Pi}(y) \\
& +\left(\frac{1}{g_{\rho}}+\theta^{2}\right)^{2} \int \frac{1}{(\rho-y)^{2}} d \mu_{\Pi}(y)+2 \theta^{4} g_{\rho}^{4} \int \frac{y}{(\rho-y)} d \mu_{\Pi}(y) \\
& +\theta^{2} g_{\rho}^{2}\left(\theta^{2} g_{\rho}^{2}+2 \theta^{2} g_{\rho}+2\right),
\end{aligned}
$$

with

$$
\begin{aligned}
& \int \frac{y}{(\rho-y)} d \mu_{\Pi}(y)=-1+\rho g_{\rho} \\
& \int \frac{y}{(\rho-y)^{2}} d \mu_{\Pi}(y)=-g_{\rho}-\rho g_{\rho}^{\prime}
\end{aligned}
$$




$$
\int \frac{y^{2}}{(\rho-y)^{2}} d \mu_{\Pi}(y)=1-2 \rho g_{\rho}-\rho^{2} g_{\rho}^{\prime},
$$

and $g_{\rho}^{\prime}=G_{\Pi}^{\prime}(\rho)=\frac{g_{\rho}\left(1-g_{\rho}\right)}{\rho\left(2 g_{\rho}-1\right)}$ (after differentiating the equation $t G_{\Pi}(t)^{2}-t G_{\pi}(t)+1=$ $0)$. Thus,

$$
\begin{gathered}
C_{\rho}^{(1)}=-\theta^{4} g_{\rho}^{4}+\frac{g_{\rho}^{\prime}}{g_{\rho}^{2}}\left(g_{\rho}+1\right), C_{\rho}^{(2)}=-2 \theta \\
v_{\rho}=-\frac{3}{5}\left(\theta^{2} g_{\rho}+2\right)^{2}-\frac{g_{\rho}^{\prime}}{g_{\rho}^{2}}\left(1+\frac{7}{g_{\rho}}+\theta^{2}\right)-4 \theta^{2} g_{\rho} .
\end{gathered}
$$

Now, set

$$
C=\left|\frac{C_{\rho}^{(2)}}{C_{\rho}^{(1)}}\right|, \sigma^{2}=\frac{v_{\rho}}{\left(C_{\rho}^{(1)}\right)^{2}} .
$$

According to Theorem 4.1. $\sqrt{N}(\lambda(N, \rho)-\rho)$ converges in distribution to the probability measure with density function

$$
f(x)=\frac{1}{2 \sqrt{6 \pi} C \sigma} \int_{-\sqrt{3} C}^{\sqrt{3} C} \exp \left(-(x-t)^{2} / 2 \sigma^{2}\right) d t .
$$

\section{$5 \quad$ Preliminary results}

\subsection{Basic bounds and convergences}

We start with straightforward bounds and convergences involving resolvents and that will be of basic use for the proof of Theorem 4.1. For any $w \in M_{m}(\mathbb{C})$ such that $w \otimes I_{N-1}-\alpha \otimes \frac{W_{N-1}}{\sqrt{N}}-\beta \otimes A_{N-1}$, resp. $w \otimes 1_{\mathcal{A}}-\alpha \otimes x-\beta \otimes a$, is invertible, define

$$
R_{N-1}(w)=\left(w \otimes I_{N-1}-\alpha \otimes \frac{W_{N-1}}{\sqrt{N}}-\beta \otimes A_{N-1}\right)^{-1},
$$

resp.

$$
R_{\infty}(w)=\left(w \otimes 1_{\mathcal{A}}-\alpha \otimes x-\beta \otimes a\right)^{-1} .
$$

Note that we have the following resolvent identities for any $w_{1}$ and $w_{2}$ in $M_{m}(\mathbb{C})$ such that the resolvents are defined:

$$
\begin{gathered}
R_{N-1}\left(w_{1}\right)-R_{N-1}\left(w_{2}\right)=R_{N-1}\left(w_{1}\right)\left[\left(w_{2}-w_{1}\right) \otimes I_{N-1}\right] R_{N-1}\left(w_{2}\right), \\
R_{\infty}\left(w_{1}\right)-R_{\infty}\left(w_{2}\right)=R_{\infty}\left(w_{1}\right)\left[\left(w_{2}-w_{1}\right) \otimes 1_{\mathcal{A}}\right] R_{\infty}\left(w_{2}\right) .
\end{gathered}
$$

Lemma 5.1. - For any $w \in M_{m}(\mathbb{C})$ such that $\Im w>0, w \otimes I_{N-1}-\alpha \otimes \frac{W_{N-1}}{\sqrt{N}}-$ $\beta \otimes A_{N-1}, w \otimes 1_{\mathcal{A}}-\alpha \otimes x-\beta \otimes a$ and $w \otimes 1_{\mathcal{A}}-\alpha \otimes x-\beta \otimes a_{N-1}$ are invertible and

$$
\begin{gathered}
\left\|R_{N-1}(w)\right\| \leq\left\|(\Im w)^{-1}\right\|, \\
\left\|R_{\infty}(w)\right\| \leq\left\|(\Im w)^{-1}\right\|, \\
\left\|\left(w \otimes 1_{\mathcal{A}}-\alpha \otimes x-\beta \otimes a_{N-1}\right)^{-1}\right\| \leq\left\|(\Im w)^{-1}\right\| .
\end{gathered}
$$


- Let $\tilde{\Omega}_{N-1}$ be as defined by (4.6) and $C_{\epsilon}$ be as in (4.7) and (4.8). Let $z$ be in $\mathbb{R}$ such that $|z-\rho|<C_{\epsilon} / 4$ and $z_{0}$ be in $\mathbb{C}$ such that $\left|z_{0}\right|<C_{\epsilon} / 4$.

Then, $\left(z e_{11}+z_{0} I_{m}-\gamma\right) \otimes 1_{\mathcal{A}}-\alpha \otimes x-\beta \otimes a,\left(z e_{11}+z_{0} I_{m}-\gamma\right) \otimes 1_{\mathcal{A}}-\alpha \otimes x-$ $\beta \otimes a_{N-1}$ and $\left(z e_{11}+z_{0} I_{m}-\gamma\right) \otimes I_{N-1}-\alpha \otimes \frac{W_{N-1}}{\sqrt{N}}-\beta \otimes A_{N-1}$ on $\tilde{\Omega}_{N-1}$, are invertible and we have

$$
\begin{gathered}
\left\|R_{N-1}\left(z e_{11}+z_{0} I_{m}-\gamma\right) \mathbf{1}_{\tilde{\Omega}_{N-1}}\right\| \leq 2 / C_{\epsilon}, \\
\left\|R_{\infty}\left(z e_{11}+z_{0} I_{m}-\gamma\right)\right\| \leq 2 / C_{\epsilon}, \\
\left\|\left(\left(z e_{11}+z_{0} I_{m}-\gamma\right) \otimes 1_{\mathcal{A}}-\alpha \otimes x-\beta \otimes a_{N-1}\right)^{-1}\right\| \leq 4 / C_{\epsilon} .
\end{gathered}
$$

Moreover, for any $t$ in the spectrum of $a, \omega_{m}\left(z e_{11}+z_{0} I_{m}-\gamma\right)-t \beta$ is invertible and

$$
\left\|\left(\omega_{m}\left(z e_{11}+z_{0} I_{m}-\gamma\right)-t \beta\right)^{-1}\right\| \leq 2 / C_{\epsilon},
$$

and, for any $t$ in the spectrum of $A_{N-1}, \omega_{m}^{(N)}\left(z e_{11}+z_{0} I_{m}-\gamma\right)-t \beta$ is invertible and

$$
\left\|\left(\omega_{m}^{(N)}\left(z e_{11}+z_{0} I_{m}-\gamma\right)-t \beta\right)^{-1}\right\| \leq 4 / C_{\epsilon} .
$$

Proof. (5.3), (5.4) and (5.5) come from Lemma 3.1 (i) of 29]. Now, according to [32, since $A_{N-1}$ (and thus $a_{N-1}$ ) converges strongly to $a$, we have, for all large $N$,

$\operatorname{spect}\left(\left(\rho e_{11}-\gamma\right) \otimes 1_{\mathcal{A}}-\alpha \otimes x-\beta \otimes a_{N-1}\right)$

$$
\left.\subset \operatorname{spect}\left(\left(\rho e_{11}-\gamma\right) \otimes 1_{\mathcal{A}}-\alpha \otimes x-\beta \otimes a\right)+\right]-C_{\epsilon} / 4, C_{\epsilon} / 4[.
$$

(5.6), (5.7) and (5.8) easily follow from (4.7), (4.8), (5.11) and the following facts: if $y$ is a self-adjoint element in a $\mathcal{C}^{*}$-algebra and $\lambda_{0} \in \mathbb{C} \backslash \operatorname{spect}(y)$, then $d\left(\lambda_{0}\right.$, spect $\left.(y)\right)=$ $1 /\left\|\left(\lambda_{0}-y\right)^{-1}\right\|$ and for any other element $\tilde{y}$, the distance between any element in the spectrum of $\tilde{y}$ and the spectrum of $y$ is smaller than $\|y-\tilde{y}\|$.

Using the analyticity on the set $\left\{w \in M_{m}(\mathbb{C}), w \otimes 1_{\mathcal{A}}-\alpha \otimes x-\beta \otimes a\right.$ invertible $\}$ of the functions $E_{\mathcal{N}}\left[R_{\infty}(\cdot)\right]$ and $\omega_{m}$, one can easily deduce from (3.6) that,

$$
\begin{aligned}
I_{m} & \otimes \\
& 1_{\mathcal{A}} \\
& =\left(\omega_{m}\left(z e_{11}+z_{0} I_{m}-\gamma\right) \otimes 1_{\mathcal{A}}-\beta \otimes a\right) E_{\mathcal{N}}\left[R_{\infty}\left(z e_{11}+z_{0} I_{m}-\gamma\right)\right] \\
& =E_{\mathcal{N}}\left[R_{\infty}\left(z e_{11}+z_{0} I_{m}-\gamma\right)\right]\left(\omega_{m}\left(z e_{11}+z_{0} I_{m}-\gamma\right) \otimes 1_{\mathcal{A}}-\beta \otimes a\right) .
\end{aligned}
$$

Let $t$ be in the spectrum of $a$. Choose a character $\chi$ of the commutative $\mathcal{C}^{*}$-algebra $\mathbb{C}\langle a\rangle$ such that $\chi(a)=t$ and denote by $\chi_{m}: M_{m}(\mathbb{C}<a>) \rightarrow M_{m}(\mathbb{C})$ the algebra homomorphism obtained by applying $\chi$ to each entry. Applying $\chi_{m}$ to (5.12), we deduce that

$$
\left(\omega_{m}\left(z e_{11}+z_{0} I_{m}-\gamma\right)-t \beta\right)^{-1}=\chi_{m}\left(E_{\mathcal{N}}\left[R_{\infty}\left(z e_{11}+z_{0} I_{m}-\gamma\right)\right]\right)
$$

so that (5.9) readily follows from (5.7). (5.10) can be proven similarly. 
The following convergence results are quite straightforward consequences of asymptotic freeness of $W_{N-1} / \sqrt{N}$ and $A_{N-1}$.

Lemma 5.2. For any $\Sigma, \Sigma_{1}, \Sigma_{2}$ in $M_{m}(\mathbb{C})$ such that $\exists C>0,\|\Sigma\| \leq C,\left\|\Sigma_{1}\right\| \leq$ $C,\left\|\Sigma_{2}\right\| \leq C$, almost surely,

1)

$$
\begin{gathered}
\left(\operatorname{id}_{m} \otimes \operatorname{tr}_{N-1}\right)\left(R_{N-1}\left(\rho_{N} e_{11}-\gamma\right)\left(\Sigma \otimes I_{N-1}\right) R_{N-1}\left(\rho_{N} e_{11}-\gamma\right)\right) \mathbf{1}_{\tilde{\Omega}_{N-1}} \\
\longrightarrow{ }_{N \rightarrow+\infty}\left(\operatorname{id}_{m} \otimes \phi\right)\left[R_{\infty}\left(\rho e_{11}-\gamma\right)\left(\Sigma \otimes 1_{\mathcal{A}}\right) R_{\infty}\left(\rho e_{11}-\gamma\right)\right]
\end{gathered}
$$

2)

$$
\begin{aligned}
\mathbf{1}_{\tilde{\Omega}_{N-1}} \times \operatorname{tr}_{N-1}\left\{\left(\operatorname{Tr}_{m} \otimes \mathrm{id}_{N-1}\right)\left[R_{N-1}\left(\rho_{N} e_{11}-\gamma\right)\left(\Sigma_{1} \otimes I_{N-1}\right)\right]\right. \\
\left.\times\left(\operatorname{Tr}_{m} \otimes \operatorname{id}_{N-1}\right)\left[R_{N-1}\left(\rho_{N} e_{11}-\gamma\right)\left(\Sigma_{2} \otimes I_{N-1}\right)\right]\right\} \\
\longrightarrow{ }_{N \rightarrow+\infty} \phi\left\{\left(\operatorname{Tr}_{m} \otimes \operatorname{id}_{\mathcal{A}}\right)\left[R_{\infty}\left(\rho e_{11}-\gamma\right)\left(\Sigma_{1} \otimes 1_{\mathcal{A}}\right)\right]\right. \\
\left.\times\left(\operatorname{Tr}_{m} \otimes \operatorname{id}_{\mathcal{A}}\right)\left[R_{\infty}\left(\rho e_{11}-\gamma\right)\left(\Sigma_{2} \otimes 1_{\mathcal{A}}\right)\right]\right\},
\end{aligned}
$$

3)

$$
\begin{gathered}
\forall w \in M_{m}(\mathbb{C}), \Im w>0, \omega_{m}^{(N)}(w) \longrightarrow_{N \rightarrow+\infty} \omega_{m}(w) . \\
\forall z \in \mathbb{R},|z-\rho|<\tau, \omega_{m}^{(N)}\left(z e_{11}-\gamma\right) \longrightarrow_{N \rightarrow+\infty} \omega_{m}\left(z e_{11}-\gamma\right) . \\
\omega_{m}^{(N)}\left(\rho_{N} e_{11}-\gamma\right) \longrightarrow{ }_{N \rightarrow+\infty} \omega_{m}\left(\rho e_{11}-\gamma\right) .
\end{gathered}
$$

Proof. We have for any self-adjoint operators $u$ and $v$, for any $w \in M_{m}(\mathbb{C})$ such that $\Im w>0$, for any non null integer $p$,

$$
\begin{aligned}
(w \otimes 1-\alpha \otimes u-\beta \otimes v)^{-1} \\
\quad \\
\quad \sum_{k=0}^{p-1} w^{-1} \otimes 1\left(\alpha w^{-1} \otimes u+\beta w^{-1} \otimes v\right)^{k} \\
\quad+(w \otimes 1-\alpha \otimes u-\beta \otimes v)^{-1}\left(\alpha w^{-1} \otimes u+\beta w^{-1} \otimes v\right)^{p} .
\end{aligned}
$$

For any $K>0$, define

$$
\mathcal{O}_{K}=\left\{w \in M_{m}(\mathbb{C}), \Im(w)>K I_{m}\right\} .
$$

According to Lemma 3.1 (i) of [29, for any $w \in \mathcal{O}_{K}$, we have $\left\|w^{-1}\right\| \leq 1 / K$. Let $0<C<1$. For any $\kappa>0$, there exists $K=K(\kappa, \alpha, \beta)>0$ such that if $w \in \mathcal{O}_{K}$, for any $u$ and $v$ such that $\|u\| \leq \kappa$ and $\|v\| \leq \kappa$ then

$$
\left\|\left(\alpha w^{-1} \otimes u+\beta w^{-1} \otimes v\right)\right\| \leq C,
$$

so that (using once more Lemma 3.1 (i) of 29])

$$
\sup _{w \in \mathcal{O}_{K}}\left\|(w \otimes 1-\alpha \otimes u-\beta \otimes v)^{-1}\left(\alpha w^{-1} \otimes u+\beta w^{-1} \otimes v\right)^{p}\right\| \leq \frac{C^{p}}{K} \rightarrow_{p \rightarrow+\infty} 0 .
$$


Fix $K>0$ such that (5.19) holds for $(u, v)=(x, a)$ and $(u, v)=\left(\frac{W_{N-1}}{\sqrt{N}}, A_{N-1}\right)$ on $\tilde{\Omega}_{N-1}$. Therefore, for any $\delta>0$, we can find a polynomial $Q_{w}$ with coefficients in $M_{m}(\mathbb{C})$ depending on $w, \alpha$ and $\beta$ such that:

$$
\begin{gathered}
\sup _{w \in \mathcal{O}_{K}}\left\|R_{\infty}(w)-Q_{w}(x, a)\right\| \leq \delta, \\
\sup _{w \in \mathcal{O}_{K}}\left\|R_{N-1}(w)-Q_{w}\left(\frac{W_{N-1}}{\sqrt{N}}, A_{N-1}\right)\right\| \mathbf{1}_{\tilde{\Omega}_{N-1}} \leq \delta .
\end{gathered}
$$

Now, by the asymptotic freeness of $\frac{W_{N-1}}{\sqrt{N}}$ and $A_{N-1}$ (see [2, Theorem 5.4.5]), we have that almost surely

$$
\begin{gathered}
\left(\operatorname{id}_{m} \otimes \operatorname{tr}_{N-1}\right)\left\{Q_{w}\left(\frac{W_{N-1}}{\sqrt{N}}, A_{N-1}\right)\left(\Sigma \otimes I_{N}\right) Q_{w}\left(\frac{W_{N-1}}{\sqrt{N}}, A_{N-1}\right)\right\} \\
\longrightarrow N \rightarrow \infty\left(\operatorname{id}_{m} \otimes \phi\right)\left\{Q_{w}(x, a)\left(\Sigma \otimes 1_{\mathcal{A}}\right) Q_{w}(x, a)\right\} .
\end{gathered}
$$

Using (5.22), (5.20), (5.21), (5.3), (5.4) and

$$
\lim _{N \rightarrow+\infty} \mathbf{1}_{\tilde{\Omega}_{N-1}}=1 \text { a.s., }
$$

we can deduce that for $w \in \mathcal{O}_{K}$, $\left(\operatorname{id}_{m} \otimes \operatorname{tr}_{N-1}\right)\left(R_{N-1}(w)\left(\Sigma \otimes I_{N-1}\right) R_{N-1}(w)\right)$ converges almost surely towards $\left(\operatorname{id}_{m} \otimes \phi\right)\left(R_{\infty}(w)\left(\Sigma \otimes 1_{\mathcal{A}}\right) R_{\infty}(w)\right)$, when $N$ goes to infinity.

Let $\mathcal{O}=\left\{w \in M_{m}(\mathbb{C}), \Im(w)>0\right\}$. The two functions

$$
\Phi_{N}(w)=\left(\operatorname{id}_{m} \otimes \operatorname{tr}_{N-1}\right)\left[R_{N-1}(w)\left(\Sigma \otimes I_{N-1}\right) R_{N-1}(w)\right]
$$

and

$$
\Phi_{\infty}(w)=\left(\operatorname{id}_{m} \otimes \phi\right)\left[R_{\infty}(w)\left(\Sigma \otimes 1_{\mathcal{A}}\right) R_{\infty}(w)\right]
$$

are holomorphic on $\mathcal{O}$. Moreover, using (5.3), we have

$$
\left\|\Phi_{N}(w)\right\| \leq\left\|(\Im w)^{-1}\right\|^{2}\|\Sigma\| \leq C\left\|(\Im w)^{-1}\right\|^{2} .
$$

It readily follows that $\Phi_{N}$ is a bounded sequence in the set of analytic functions on $\mathcal{O}$ endowed with the uniform convergence on compact subsets. Since moreover, almost surely, for any $t>K, t \in \mathbb{Q}, \Phi_{N}\left(i t I_{m}\right)$ converges towards $\Phi\left(i t I_{m}\right)$, we can apply Vitali's theorem to conclude that almost surely the convergence of $\Phi_{N}$ towards $\Phi_{\infty}$ holds on $\mathcal{O}$. Of course, this convergence still holds on $-\mathcal{O}$.

Let $z \in \mathbb{R}$ be such that $|z-\rho| \leq C_{\epsilon} / 4$. For any $q>0$, such that $\frac{1}{q} \leq C_{\epsilon} / 4$, define $w(q)=z e_{11}-\gamma+i \frac{1}{q} I_{m}$. Almost surely, for any such $q, \Phi_{N}(w(q))$ converges towards $\Phi_{\infty}(w(q))$. Using (5.23), the resolvent identities (5.2) and (5.1) on $\tilde{\Omega}_{N-1}$, and the bounds (5.6) and (5.7), we easily deduce by letting q goes to infinity that a.s.

$$
\begin{aligned}
& \left(\operatorname{id}_{m} \otimes \operatorname{tr}_{N-1}\right)\left[R_{N-1}\left(z e_{11}-\gamma\right)\left(\Sigma \otimes I_{N-1}\right) R_{N-1}\left(z e_{11}-\gamma\right)\right] \mathbf{1}_{\tilde{\Omega}_{N-1}} \\
& \longrightarrow N \rightarrow+\infty\left(\operatorname{id}_{m} \otimes \phi\right)\left[R_{\infty}\left(z e_{11}-\gamma\right)\left(\Sigma \otimes 1_{\mathcal{A}}\right) R_{\infty}\left(z e_{11}-\gamma\right)\right] .
\end{aligned}
$$

Note that using (4.16), the bound (5.6), and the resolvent identity (5.1) on $\tilde{\Omega}_{N-1}$, (5.13) readily follows from (5.24) applied to $z=\rho$.

(5.14), (5.15), (5.16) and (5.17) can be proven using similar ideas. 
The proof of Theorem 4.1, that will be presented in Section [6, is based on the writing of the outlier in terms of a quadratic form involving the resolvent $R_{N-1}$. Section 5.2 presents the central limit theorem for random quadratic forms involved in the proof whereas Section 5.3 gather results that will be used to prove that some terms are negligible.

\subsection{Central limit theorem for random quadratic forms}

Proposition 5.1. For any Hermitian $m \times m$ matrix $H$,

$$
\begin{aligned}
& \sqrt{N}\left(\frac{1}{N} \operatorname{Tr}_{m}\left\{H\left(\alpha \otimes Y^{*}\right) R_{N-1}\left(\rho_{N} e_{11}-\gamma\right) \mathbf{1}_{\tilde{\Omega}_{N-1}}(\alpha \otimes Y)\right\}\right. \\
& \left.-\frac{1}{N} \operatorname{Tr}_{m}\left\{\alpha H \alpha\left[\left(\operatorname{id}_{m} \otimes \operatorname{Tr}_{N-1}\right)\left(R_{N-1}\left(\rho_{N} e_{11}-\gamma\right) \mathbf{1}_{\tilde{\Omega}_{N-1}}\right)\right]\right\}\right)
\end{aligned}
$$

converges in distribution to a Gaussian variable with mean 0 and variance

$$
\begin{gathered}
\left(\mathbb{E}\left(\left|W_{12}\right|^{4}\right)-2\right) \int\left[\operatorname{Tr}_{m}\left(\alpha H \alpha\left(\omega_{m}\left(\rho e_{11}-\gamma\right)-t \beta\right)^{-1}\right)\right]^{2} d \mu_{a}(t) \\
\left.+\phi\left(\left[\left(\operatorname{Tr}_{m} \otimes \operatorname{id}_{\mathcal{A}}\right)\left\{\left(\rho e_{11}-\gamma\right) \otimes 1_{\mathcal{A}}-\alpha \otimes x-\beta \otimes a\right)^{-1}\left((\alpha H \alpha) \otimes 1_{\mathcal{A}}\right)\right\}\right]^{2}\right) .
\end{gathered}
$$

Proof. We apply the following Proposition 5.2 to $B=R_{N-1}\left(\rho_{N} e_{11}-\gamma\right) \mathbf{1}_{\tilde{\Omega}_{N-1}}$ by using (4.16), (5.6) and Proposition 5.4 below.

Proposition 5.2. Let $m$ be a fixed integer number and $\alpha$ be a Hermitian $m \times m$ deterministic matrix that does not depend on $N$. Let $B$ be a random Hermitian $m N \times m N$ matrix such that there exists $C>0$ such that $\|B\| \leq C$. Let us write $B=\sum_{i, j=1}^{N} B_{i j} \otimes E_{i j}$ where $B_{i j}$ are $m \times m$ matrices. Assume that, for any $p, q, p^{\prime}, q^{\prime}$ in $\{1, \ldots, m\}^{4}$,

- $\frac{1}{N} \sum_{i=1}^{N}\left(B_{i i}\right)_{p q}\left(B_{i i}\right)_{p^{\prime} q^{\prime}} \rightarrow_{N \rightarrow+\infty} \omega_{(p, q),\left(p^{\prime}, q^{\prime}\right)}$ in probability,

- $\frac{1}{N} \sum_{i, j=1}^{N}\left(B_{i j}\right)_{p q}\left(B_{j i}\right)_{p^{\prime} q^{\prime}} \rightarrow_{N \rightarrow+\infty} \theta_{(p, q),\left(p^{\prime}, q^{\prime}\right)}$ in probability.

Let ${ }^{t} X=\left(x_{1}, \ldots, x_{N}\right)$ be an independent vector of size $N$ which contains i.i.d. complex standardized entries with bounded fourth moment and such that $\mathbb{E}\left(x_{1}^{2}\right)=0$. Let $H$ be a $m \times m$ deterministic Hermitian matrix that does not depend on $N$. Then, when $N$ goes to infinity,

$$
\frac{1}{\sqrt{N}} \operatorname{Tr}_{m}\left\{H\left[\left(\alpha \otimes X^{*}\right) B(\alpha \otimes X)-\alpha\left(\mathrm{id}_{m} \otimes \operatorname{Tr}_{N}\right)(B) \alpha\right]\right\}
$$

converges in distribution to a Gaussian variable with mean 0 and variance

$$
\begin{gathered}
\left(\mathbb{E}\left(\left|x_{1}\right|^{4}\right)-2\right) \sum_{p, q, p^{\prime}, q^{\prime}=1}^{m}(\alpha H \alpha)_{q p}(\alpha H \alpha)_{q^{\prime} p^{\prime}} \omega_{(p, q),\left(p^{\prime}, q^{\prime}\right)} \\
+\sum_{p, q, p^{\prime}, q^{\prime}=1}^{m}(\alpha H \alpha)_{q p}(\alpha H \alpha)_{q^{\prime} p^{\prime}} \theta_{(p, q),\left(p^{\prime}, q^{\prime}\right)} .
\end{gathered}
$$


Proof. Note that

$\frac{1}{\sqrt{N}} \operatorname{Tr}_{m}\left\{H\left[\left(\alpha \otimes X^{*}\right) B(\alpha \otimes X)-\alpha\left(\operatorname{id}_{m} \otimes \operatorname{Tr}_{N}\right)(B) \alpha\right]\right\}=\frac{1}{\sqrt{N}}\left\{X^{*} \mathcal{B} X-\operatorname{Tr}_{N} \mathcal{B}\right\}$

where $\mathcal{B}=\left(\mathcal{B}_{i j}\right)_{1<i, j \leq N}$ and $\mathcal{B}_{i j}=\operatorname{Tr}_{m} \alpha H \alpha B_{i j}$.

Thus, the result follows from [6] or Theorem 5.2 in [20].

Proposition 5.3. When it is defined, let us rewrite

$$
R_{N-1}=\sum_{i, j=1}^{N-1}\left(R_{N-1}\right)_{i j} \otimes E_{i j},
$$

where $\left(R_{N-1}\right)_{i j} \in M_{m}(\mathbb{C})$. For any $w \in \mathbb{H}_{m}^{+}(\mathbb{C})$, we have that, almost surely,

$$
\begin{gathered}
F_{N}(w)=\frac{1}{N-1} \sum_{i=1}^{N-1}\left[\left(R_{N-1}(w-\gamma)\right)_{i i}\right]_{p q}\left[\left(R_{N-1}(w-\gamma)_{i i}\right)\right]_{p^{\prime} q^{\prime}} \\
\rightarrow_{N \rightarrow+\infty} \int\left[\left(\omega_{m}(w-\gamma)-t \beta\right)^{-1}\right]_{p q}\left[\left(\omega_{m}(w-\gamma)-t \beta\right)^{-1}\right]_{p^{\prime} q^{\prime}} d \mu_{a}(t)
\end{gathered}
$$

Proof. First we are going to prove that almost surely,

$$
\begin{aligned}
& \frac{1}{N-1} \sum_{i=1}^{N-1}\left[\left(R_{N-1}(w-\gamma)\right)_{i i}\right]_{p q}\left[\left(R_{N-1}(w-\gamma)\right)_{i i}\right]_{p^{\prime} q^{\prime}} \\
& -\frac{1}{N-1} \sum_{i=1}^{N-1}\left[\mathbb{E}\left(R_{N-1}(w-\gamma)\right)_{i i}\right]_{p q}\left[\mathbb{E}\left(R_{N-1}(w-\gamma)\right)_{i i}\right]_{p^{\prime} q^{\prime}} \longrightarrow_{N \rightarrow+\infty} 0 .
\end{aligned}
$$

Set $a_{i}=\left[\left(R_{N-1}(w-\gamma)\right)_{i i}\right]_{p q}$ and $b_{i}=\left[\left(R_{N-1}(w-\gamma)\right)_{i i}\right]_{p^{\prime} q^{\prime}}$. We have

$$
\begin{aligned}
\frac{1}{N-1} \sum_{i=1}^{N-1} a_{i} b_{i}- & \frac{1}{N-1} \sum_{i=1}^{N-1} \mathbb{E}\left(a_{i}\right) \mathbb{E}\left(b_{i}\right) \\
= & \frac{1}{N-1} \sum_{i=1}^{N-1} a_{i} b_{i}-\frac{1}{N-1} \sum_{i=1}^{N-1} \mathbb{E}\left(a_{i} b_{i}\right) \\
& +\frac{1}{N-1} \sum_{i=1}^{N-1} \mathbb{E}\left\{\left(a_{i}-\mathbb{E}\left(a_{i}\right)\right)\left(b_{i}-\mathbb{E}\left(b_{i}\right)\right)\right\} .
\end{aligned}
$$

Consider the linear isomorphism $\Psi$ between $M_{N-1}^{s a}(\mathbb{C})$ and $\mathbb{R}^{(N-1)^{2}}$ given by

$$
\Psi\left(\left(a_{k l}\right)_{1 \leq k, l \leq N-1}\right)=\left(\left(a_{k k}\right)_{1 \leq k \leq N-1},\left(\sqrt{2} \Re a_{k l}\right)_{1 \leq k<l \leq N-1},\left(\sqrt{2} \Im a_{k l}\right)_{1 \leq k<l \leq N-1}\right)
$$


for $\left(a_{k l}\right)_{1 \leq k, l \leq N-1}$ in $M_{N-1}^{s a}(\mathbb{C}) . M_{N-1}(\mathbb{C})^{s a}$ is an Euclidean space with inner product given by $\langle A, B\rangle=\operatorname{Tr}_{N-1}(A B)$ and with norm

$$
\|A\|_{e}=\left(\operatorname{Tr}_{N-1} A^{2}\right)^{1 / 2} .
$$

We shall identify $M_{N-1}^{s a}(\mathbb{C})$ with $R^{(N-1)^{2}}$ via the isomorphism $\Psi$. Note that under this identification the norm $\|\cdot\|_{e}$ on $M_{N-1}^{s a}(\mathbb{C})$ corresponds to the usual Euclidean norm on $R^{(N-1)^{2}}$.

Define $f_{N}: M_{N-1}^{s a}(\mathbb{C}) \mapsto \mathbb{C}$ by

$$
\begin{aligned}
f_{N}(W) & =\frac{1}{N-1} \sum_{i=1}^{N-1} \operatorname{Tr}_{m(N-1)}\left[\left((w-\gamma) \otimes I_{N-1}-\alpha \otimes W-\beta \otimes A_{N-1}\right)^{-1}\left(e_{q p} \otimes E_{i i}\right)\right] \\
& \times \operatorname{Tr}_{m(N-1)}\left[\left((w-\gamma) \otimes I_{N-1}-\alpha \otimes W-\beta \otimes A_{N-1}\right)^{-1}\left(e_{q^{\prime} p^{\prime}} \otimes E_{i i}\right)\right] .
\end{aligned}
$$

Using the resolvent identity, for $H_{1}, H_{2} \in M_{m(N-1)}^{s a}(\mathbb{C})$,

$$
\begin{aligned}
\left(w \otimes I_{N-1}-H_{1}\right)^{-1}-\left(w \otimes I_{N-1}-H_{2}\right)^{-1} & \\
& =\left(w \otimes I_{N-1}-H_{1}\right)^{-1}\left(H_{1}-H_{2}\right)\left(w \otimes I_{N-1}-H_{2}\right)^{-1},
\end{aligned}
$$

and [29. Lemma 3.1 (i)], one can easily prove that $f_{N} \circ \Psi^{-1}$ is Lipschitz with constant $\left\|(\Im w)^{-1}\right\|^{3}$. Therefore, according to Lemma A.1.

$$
\mathbb{P}\left(\left|\frac{1}{N-1} \sum_{i=1}^{N-1}\left(a_{i} b_{i}-\mathbb{E}\left(a_{i} b_{i}\right)\right)\right|>\varepsilon\right) \leq K_{1} \exp \left(-K_{2} N^{1 / 2}\left\|(\Im w)^{-1}\right\|^{-3} \varepsilon\right) .
$$

By Borell-Cantelli lemma, we deduce that, almost surely, when $N$ goes to infinity, $\frac{1}{N-1} \sum_{i=1}^{N-1} a_{i} b_{i}-\frac{1}{N-1} \sum_{i=1}^{N-1} \mathbb{E}\left(a_{i} b_{i}\right)$ goes to zero.

Now define $g_{N}: M_{N-1}^{s a}(\mathbb{C}) \mapsto \mathbb{C}$ by

$$
g_{N}(W)=\operatorname{Tr}_{m(N-1)}\left[\left((w-\gamma) \otimes I_{N-1}-\alpha \otimes W-\beta \otimes A_{N-1}\right)^{-1}\left(e_{q p} \otimes E_{i i}\right)\right] .
$$

Define also $\tilde{g}_{N}: \mathbb{R}^{(N-1)^{2}} \rightarrow \mathbb{C}$ by $\tilde{g}_{N}=g_{N} \circ \Psi^{-1}$, where $\Psi$ is defined in (5.28). Note that

$$
\left\|\nabla \tilde{g}_{N}(\Psi(W))\right\|=\left\|\operatorname{grad} g_{N}(W)\right\|_{e}
$$

and

$$
\left\|\operatorname{grad} g_{N}(W)\right\|_{e}^{2}=\sup _{w \in S_{1}\left(M_{N-1}^{s a}(\mathbb{C})\right)}\left|\frac{d}{d t} g_{N}(W+t w)_{\mid t=0}\right|^{2},
$$

where $S_{1}\left(M_{N-1}^{s a}(\mathbb{C})\right)$ denotes the unit sphere of $M_{N-1}^{s a}(\mathbb{C})$ with respect to $\|\cdot\|_{e}$. Applying Poincaré inequality for $\tilde{g}_{N}$, we get that

$$
\mathbb{E}\left(\left|g_{N}\left(\frac{W_{N-1}}{\sqrt{N}}\right)-\mathbb{E}\left\{g_{N}\left(\frac{W_{N-1}}{\sqrt{N}}\right)\right\}\right|^{2}\right) \leq \frac{C}{N} \mathbb{E}\left(\left\|\operatorname{grad} g_{N}\left(\frac{W_{N-1}}{\sqrt{N}}\right)\right\|_{e}^{2}\right) .
$$


Using (5.29) and (5.3), it readily follows that, there exists $C>0$, such that for any $i=1, \ldots, N-1$,

$$
\mathbb{E}\left|a_{i}-\mathbb{E}\left(a_{i}\right)\right|^{2} \leq \frac{C\left\|(\Im w)^{-1}\right\|^{4}}{N}
$$

and similarly

$$
\mathbb{E}\left|b_{i}-\mathbb{E}\left(b_{i}\right)\right|^{2} \leq \frac{C\left\|(\Im w)^{-1}\right\|^{4}}{N}
$$

so that $\frac{1}{N-1} \sum_{i=1}^{N-1} \mathbb{E}\left\{\left(a_{i}-\mathbb{E}\left(a_{i}\right)\right)\left(b_{i}-\mathbb{E}\left(b_{i}\right)\right)\right\}$ goes to zero as $N$ goes to infinity. Thus, the proof of (5.27) is complete.

Lemma 5.3. For any $w \in \mathbb{H}_{m}^{+}(\mathbb{C})$, for any $j \in\{1, \ldots, N-1\}$,

$$
\mathbb{E}\left\{\left(R_{N-1}(w-\gamma)\right)_{j j}\right\}=\left(\omega_{m}^{(N)}(w-\gamma)-d_{j} \beta\right)^{-1}+O_{j}^{(u)}(1 / \sqrt{N}) .
$$

Proof. First set

$$
\hat{R}_{N-1}(w)=\left(w \otimes I_{N-1}-\alpha \otimes \frac{W_{N-1}}{\sqrt{N-1}}-\beta \otimes A_{N-1}\right)^{-1} .
$$

Using Lemma 3.1 (i) of 29], we have

$$
\left\|\hat{R}_{N-1}(w)\right\| \leq\left\|(\Im w)^{-1}\right\| .
$$

Note that,

$$
\begin{aligned}
R_{N-1}(w)= & \hat{R}_{N-1}(w) \\
& +\frac{1}{\sqrt{N-1}(\sqrt{N}+\sqrt{N-1})} \\
& \quad \quad \quad\left(I_{m} \otimes I_{N-1}-R_{N-1}(w)\left(w \otimes I_{N-1}-\beta \otimes A_{N-1}\right)\right) \hat{R}_{N-1}(w) .
\end{aligned}
$$

Thus, using (5.30), (5.3) and (4.1), it readily follows that for any $w \in \mathbb{H}_{m}^{+}(\mathbb{C})$, for any $j \in\{1, \ldots, N-1\}$,

$$
\mathbb{E}\left\{\left(R_{N-1}(w-\gamma)\right)_{j j}\right\}=\mathbb{E}\left\{\left(\hat{R}_{N-1}(w-\gamma)\right)_{j j}\right\}+O_{j}^{(u)}(1 / N) .
$$

Therefore, in the following, we will prove that

$$
\mathbb{E}\left\{\left(\hat{R}_{N-1}(w-\gamma)\right)_{j j}\right\}=\left(\omega_{m}^{(N)}(w-\gamma)-d_{j} \beta\right)^{-1}+O_{j}^{(u)}(1 / \sqrt{N}) .
$$

Denote by $\kappa_{3}$ the classical third cumulant of $\mu$. According to Corollary 5.5 in [11, for any $j \in\{1, \ldots, N-1\}$,

$$
\begin{aligned}
& \mathbb{E}\left\{\left(\hat{R}_{N-1}(w-\gamma)\right)_{j j}\right\} \\
& =\left(Y_{N-1}(w)\right)_{j j} \\
& \quad+\sum_{i, l=1}^{N-1} \frac{\kappa_{3}(1-\sqrt{-1})}{2 \sqrt{2}(N-1) \sqrt{N-1}}\left(Y_{N-1}(w)\right)_{j l} \alpha\left(Y_{N-1}(w)\right)_{i i} \alpha\left(Y_{N-1}(w)\right)_{l l} \alpha \\
& \quad \times \mathbb{E}\left\{\left(\hat{R}_{N-1}(w-\gamma)\right)_{i j}\right\}+O_{j}^{(u)}(1 / N),
\end{aligned}
$$


where

$$
Y_{N-1}(w)=\left(\left(w-\gamma-\alpha G_{N-1}(w) \alpha\right) \otimes I_{N-1}-\beta \otimes A_{N-1}\right)^{-1}
$$

with

$$
G_{N-1}(w)=\left(i d_{m} \otimes \operatorname{tr}_{N-1}\right)\left(\hat{R}_{N-1}(w-\gamma)\right)
$$

Note that according to [11, (5.7)], $\Im\left[\left(w-\gamma-\alpha G_{N-1}(w) \alpha\right)\right] \geq \Im w$ so that, indeed, by Lemma 3.1 of [29], $\left(w-\gamma-\alpha G_{N-1}(w) \alpha\right) \otimes I_{N-1}-\beta \otimes A_{N-1}$ is invertible and we have

$$
\left\|Y_{N-1}(w)\right\| \leq\left\|(\Im w)^{-1}\right\|
$$

Now set

$$
\tilde{G}_{N-1}(w)=i d_{m} \otimes \phi\left((w-\gamma) \otimes 1_{\mathcal{A}}-\alpha \otimes x-\beta \otimes a_{N-1}\right)^{-1} .
$$

Similarly, $\left(w-\gamma-\alpha \tilde{G}_{N-1}(w) \alpha\right) \otimes I_{N-1}-\beta \otimes A_{N-1}$ is invertible, we can define

$$
\tilde{Y}_{N-1}(w)=\left(\left(w-\gamma-\alpha \tilde{G}_{N-1}(w) \alpha\right) \otimes I_{N-1}-\beta \otimes A_{N-1}\right)^{-1}
$$

and we have

$$
\left\|\tilde{Y}_{N-1}(w)\right\| \leq\left\|(\Im w)^{-1}\right\| .
$$

Using the resolvent identity, (5.33), (5.36) and [11, (5.48)], one can easily deduce that there exists a polynomial $Q$ with nonnegative coefficients such that, for any $w \in M_{m}(\mathbb{C})$ such that $\Im w>0$,

$$
\left\|Y_{N-1}(w)-\tilde{Y}_{N-1}(w)\right\| \leq \frac{Q\left(\left\|(\Im w)^{-1}\right\|\right.}{\sqrt{N}} .
$$

Note that $\tilde{Y}_{N-1}(w)=\left(\omega_{m}^{(N)}(w-\gamma) \otimes I_{N-1}-\beta \otimes A_{N-1}\right)^{-1}$. Now

$$
\begin{aligned}
& \left\|\sum_{i, l=1}^{N-1} \frac{\kappa_{3}(1-\sqrt{-1})}{2 \sqrt{2}(N-1) \sqrt{N-1}}\left(Y_{N-1}\right)_{j l} \alpha\left(Y_{N-1}\right)_{i i} \alpha\left(Y_{N-1}\right)_{l l} \alpha \mathbb{E}\left\{\left(\hat{R}_{N-1}(w-\gamma)\right)_{i j}\right\}\right\| \\
& \leq \frac{C\left\|(\Im w)^{-1}\right\|^{2}}{\sqrt{N}}\left(\sum_{l=1}^{N-1}\left\|\left(Y_{N-1}\right)_{j l}\right\|^{2}\right)^{1 / 2}\left(\sum_{i=1}^{N-1}\left\|\mathbb{E}\left\{\left(\hat{R}_{N-1}(w-\gamma)\right)_{i j}\right\}\right\|^{2}\right)^{1 / 2} \\
& \leq \frac{C m\left\|(\Im w)^{-1}\right\|^{2}}{\sqrt{N}}\left\|Y_{N-1}\right\|\left\|\mathbb{E}\left(\hat{R}_{N-1}(w-\gamma)\right)\right\| \\
& \leq \frac{C m\left\|(\Im w)^{-1}\right\|^{4}}{\sqrt{N}}
\end{aligned}
$$

where we used [11, Lemma 8.1], (5.33) and (5.3). It readily follows that, for any $j \in\{1, \ldots, N-1\}$,

$$
\begin{aligned}
\mathbb{E}\left\{\left(\hat{R}_{N-1}(w-\gamma)\right)_{j j}\right\}= & \left(\left(\omega_{m}^{(N)}(w-\gamma) \otimes I_{N-1}-\beta \otimes A_{N-1}\right)^{-1}\right)_{j j} \\
& +O_{j}^{(u)}(1 / \sqrt{N}) .
\end{aligned}
$$


Now, note that there exist two permutation matrices $\Pi_{1}$ and $\Pi_{2}$ in $M_{(N-1) m}(\mathbb{C})$ such that, for any matrices $A \in M_{m}(\mathbb{C}), B \in M_{N-1}(\mathbb{C}), A \otimes B=\Pi_{1}(B \otimes A) \Pi_{2}$. Therefore

$$
\begin{aligned}
& {\left[\left(\left(\omega_{m}^{(N)}(w-\gamma) \otimes I_{N-1}-\beta \otimes A_{N-1}\right)^{-1}\right)_{j j}\right]_{p q} } \\
= & \operatorname{Tr}_{m(N-1)}\left[\left(\omega_{m}^{(N)}(w-\gamma) \otimes I_{N-1}-\beta \otimes A_{N-1}\right)^{-1}\left(e_{q p} \otimes E_{j j}\right)\right] \\
= & \operatorname{Tr}_{m(N-1)}\left[\Pi_{2}^{-1}\left(I_{N-1} \otimes \omega_{m}^{(N)}(w-\gamma)-A_{N-1} \otimes \beta\right)^{-1} \Pi_{1}^{-1} \Pi_{1}\left(E_{j j} \otimes e_{q p}\right) \Pi_{2}\right] \\
= & \operatorname{Tr}_{m(N-1)}\left[\left(I_{N-1} \otimes \omega_{m}^{(N)}(w-\gamma)-A_{N-1} \otimes \beta\right)^{-1}\left(E_{j j} \otimes e_{q p}\right)\right] \\
= & {\left[\left(\omega_{m}^{(N)}(w-\gamma)-d_{j} \beta\right)^{-1}\right]_{p q} . }
\end{aligned}
$$

Thus,

$$
\mathbb{E}\left\{\left(\hat{R}_{N-1}(w-\gamma)\right)_{j j}\right\}=\left(\omega_{m}^{(N)}(w-\gamma)-d_{j} \beta\right)^{-1}+O_{j}^{(u)}(1 / \sqrt{N}) .
$$

Lemma 5.3 follows.

Note that, using (3.4) and [29, Lemma 3.1 (i)], we have that for any $w \in H_{m}^{+}(\mathbb{C})$

$$
\begin{gathered}
\left\|\left(\omega_{m}^{(N)}(w)-d_{i} \beta\right)^{-1}\right\| \leq\left\|(\Im w)^{-1}\right\|, \\
\left\|\left(\omega_{m}(w)-d_{i} \beta\right)^{-1}\right\| \leq\left\|(\Im w)^{-1}\right\|,
\end{gathered}
$$

and then

$$
\begin{aligned}
\left\|\left(\omega_{m}^{(N)}(w)-d_{i} \beta\right)^{-1}-\left(\omega_{m}(w)-d_{i} \beta\right)^{-1}\right\| \\
\leq\left\|\left(\omega_{m}^{(N)}(w)-d_{i} \beta\right)^{-1}\left[\omega_{m}(w)-\omega_{m}^{(N)}(w)\right]\left(\omega_{m}(w)-d_{i} \beta\right)^{-1}\right\| \\
\leq\left\|(\Im w)^{-1}\right\|^{2}\left\|\omega_{m}^{(N)}(w)-\omega_{m}(w)\right\| .
\end{aligned}
$$

Recall that $F_{N}$ was defined by (5.25). Lemma 5.3 and (5.27) yield that for any $w \in H_{m}^{+}(\mathbb{C})$, almost surely,

$$
\begin{aligned}
F_{N}(w) & =\frac{1}{N-1} \sum_{i=1}^{N-1}\left[\left(\omega_{m}^{(N)}(w-\gamma)-d_{i} \beta\right)^{-1}\right]_{p q}\left[\left(\omega_{m}^{(N)}(w-\gamma)-d_{i} \beta\right)^{-1}\right]_{p^{\prime} q^{\prime}}+o(1) \\
& =\frac{1}{N-1} \sum_{i=1}^{N-1}\left[\left(\omega_{m}(w-\gamma)-d_{i} \beta\right)^{-1}\right]_{p q}\left[\left(\omega_{m}(w-\gamma)-d_{i} \beta\right)^{-1}\right]_{p^{\prime} q^{\prime}}+o(1),
\end{aligned}
$$


using (5.37) in the first line and (5.37), (5.38), (5.39) and (5.15) in the last line. Thus

$$
F_{N}(w)=\int\left[\left(\omega_{m}(w-\gamma)-t \beta\right)^{-1}\right]_{p q}\left[\left(\omega_{m}(w-\gamma)-t \beta\right)^{-1}\right]_{p^{\prime} q^{\prime}} d \mu_{A_{N-1}}(t)+o(1)
$$

where $\mu_{A_{N-1}}=\frac{1}{N-1} \sum_{i=1}^{N-1} \delta_{\lambda_{i}\left(A_{N-1}\right)}$ is the empirical spectral measure of $A_{N-1}$. Since $\mu_{A_{N-1}}$ weakly converges towards $\mu_{a}$, Proposition 5.3 follows.

Proposition 5.4. When it is defined, let us rewrite

$$
R_{N-1}=\sum_{i, j=1}^{N-1}\left(R_{N-1}\right)_{i j} \otimes E_{i j},
$$

where $\left(R_{N-1}\right)_{i j} \in M_{m}(\mathbb{C})$. We have that, almost surely,

$$
\begin{array}{r}
\left.\frac{1}{N-1} \sum_{i=1}^{N-1}\left[\left(R_{N-1}\left(\rho_{N} e_{11}-\gamma\right)\right)_{i i}\right]_{p q}\left[\left(R_{N-1}\left(\rho_{N} e_{11}-\gamma\right)\right)_{i i}\right)\right]_{p^{\prime} q^{\prime}} \mathbf{1}_{\tilde{\Omega}_{N-1}} \\
\rightarrow_{N \rightarrow+\infty} \int\left[\left(\omega_{m}\left(\rho e_{11}-\gamma\right)-t \beta\right)^{-1}\right]_{p q}\left[\left(\omega_{m}\left(\rho e_{11}-\gamma\right)-t \beta\right)^{-1}\right]_{p^{\prime} q^{\prime}} d \mu_{a}(t)
\end{array}
$$

and

$$
\begin{gathered}
\frac{1}{N-1} \sum_{i, j=1}^{N-1}\left[\left(R_{N-1}\left(\rho_{N} e_{11}-\gamma\right)\right)_{i j}\right]_{p q}\left[\left(R_{N-1}\left(\rho_{N} e_{11}-\gamma\right)\right)_{j i}\right]_{p^{\prime} q^{\prime}} \mathbf{1}_{\tilde{\Omega}_{N-1}} \rightarrow_{N \rightarrow+\infty} \\
\phi\left\{\left(\operatorname{Tr}_{m} \otimes \operatorname{id}_{\mathcal{A}}\right)\left[R_{\infty}\left(\rho e_{11}-\gamma\right)\left(e_{q p} \otimes 1_{\mathcal{A}}\right)\right]\left(\operatorname{Tr}_{m} \otimes \operatorname{id}_{\mathcal{A}}\right)\left[R_{\infty}\left(\rho e_{11}-\gamma\right)\left(e_{q^{\prime} p^{\prime}} \otimes 1_{\mathcal{A}}\right)\right]\right\} .
\end{gathered}
$$

Proof. First, with $w=\rho_{N} e_{11}-\gamma$, let us rewrite

$$
\begin{gathered}
\frac{1}{N-1} \sum_{i, j=1}^{N-1}\left\{\left[R_{N-1}(w)\right]_{i j}\right\}_{p q}\left\{\left[R_{N-1}(w)\right]_{j i}\right\}_{p^{\prime} q^{\prime}}= \\
\operatorname{tr}_{N-1}\left\{\left(\operatorname{Tr}_{m} \otimes \operatorname{id}_{N-1}\right)\left[R_{N-1}(w)\left(e_{q p} \otimes I_{N-1}\right)\right]\left(\operatorname{Tr}_{m} \otimes \operatorname{id}_{N-1}\right)\left[R_{N-1}(w)\left(e_{q^{\prime} p^{\prime}} \otimes I_{N-1}\right)\right]\right\} .
\end{gathered}
$$

Thus (5.41) readily follows from Lemma 5.2

Now, according to Lemma 5.1, on $\tilde{\Omega}_{N-1}, F_{N}$ defined by (5.25) is well defined at the points $w=z e_{11}, z e_{11}+i \frac{1}{r}$, for any $r \in \mathbb{Q} \backslash\{0\}, 0<1 / r<\tau$ and any $z \in \mathbb{R}$ such that $|z-\rho|<\tau$. Using the bounds (5.6), (5.7), (5.9) and the resolvent identities (5.1), (5.2), one can easily prove that 


$$
\begin{aligned}
& \left|F_{N}\left(z e_{11}\right) \mathbf{1}_{\tilde{\Omega}_{N-1}}-\int\left[\left(\omega_{m}\left(z e_{11}-\gamma\right)-t \beta\right)^{-1}\right]_{p q}\left[\left(\omega_{m}\left(z e_{11}-\gamma\right)-t \beta\right)^{-1}\right]_{p^{\prime} q^{\prime}} d \mu_{a}(t)\right| \\
& \leq \frac{1}{r} \frac{32}{C_{\epsilon}^{3}}\left\{1+\frac{2}{C_{\epsilon}^{2}}\|\alpha\|^{2}\right\}+\frac{4}{C_{\epsilon}^{2}} \mathbf{1}_{c_{\Omega_{N-1}}} \\
& +\mid F_{N}\left(z e_{11}+i \frac{1}{r} I_{m}\right) \\
& -\int\left[\left(\omega_{m}\left(z e_{11}+i \frac{1}{r} I_{m}-\gamma\right)-t \beta\right)^{-1}\right]_{p q} \\
& \times\left[\left(\omega_{m}\left(z e_{11}+i \frac{1}{r} I_{m}-\gamma\right)-t \beta\right)^{-1}\right]_{p^{\prime} q^{\prime}} d \mu_{a}(t) \mid .
\end{aligned}
$$

We deduce by letting $N$ go to infinity, using Proposition 5.3, and then $r$ go to infinity that for any $z \in \mathbb{R}$ such that for $|z-\rho|<\tau$, almost surely, $F_{N}\left(z e_{11}\right) \mathbf{1}_{\tilde{\Omega}_{N-1}}$ converges to $\int\left[\left(\omega_{m}\left(z e_{11}-\gamma\right)-t \beta\right)^{-1}\right]_{p q}\left[\left(\omega_{m}\left(z e_{11}-\gamma\right)-t \beta\right)^{-1}\right]_{p^{\prime} q^{\prime}} d \mu_{a}(t)$ when $N$ goes to infinity.

Note that using (4.16), the resolvent identity (5.1) on $\tilde{\Omega}_{N-1}$, and the bound (5.6), (5.40) follows from the result for $\rho$ instead of $\rho_{N}$. The proof of Proposition 5.4 is complete.

\subsection{Basic technical results of negligeability}

Lemma 5.4. For any $N$, let $X_{N}=\left(\begin{array}{c}x_{1} \\ \vdots \\ x_{N}\end{array}\right)$ be random in $\mathbb{C}^{N}$ with iid standardized entries $\left(\mathbb{E}\left(x_{i}\right)=0, \mathbb{E}\left(\left|x_{i}\right|^{2}\right)=1, \mathbb{E}\left(x_{i}^{2}\right)=0\right.$ ) and $\mathbb{E}\left(\left|x_{i}\right|^{4}\right)<\infty$. Let $m$ be a fixed integer number and $\alpha$ be a Hermitian $m \times m$ deterministic matrix. Let $B$ be a Hermitian $m N \times m N$ independent matrix such that $\sup _{N}\|B\| \leq C$. Then

$$
\frac{1}{N}\left(I_{m} \otimes X_{N}^{*}\right) B\left(I_{m} \otimes X_{N}\right)-\left(\operatorname{id}_{m} \otimes \operatorname{tr}_{N}\right) B=o_{\mathbb{P}}(1) .
$$

Proof. Let us write $B=\sum_{p, q=1}^{m} e_{p q} \otimes B^{(p q)}$ where $B^{(p q)}$ are $N \times N$ matrices. Noting that

$$
\begin{aligned}
\frac{1}{N}\left(I_{m} \otimes X_{N}^{*}\right) B & \left(I_{m} \otimes X_{N}\right)-\operatorname{id}_{m} \otimes \operatorname{tr}_{N} B \\
& =\frac{1}{N} \sum_{p, q=1}^{m} e_{p q}\left\{X_{N}^{*} B^{(p q)} X_{N}-\operatorname{Tr}_{N}\left(B^{(p q)}\right)\right\},
\end{aligned}
$$

the result readily follows from Lemma 2.7 in [4].

Lemma 5.5. For any $w \in H_{m}^{+}(\mathbb{C})$,

$$
\begin{aligned}
& \left(\operatorname{id}_{m} \otimes \operatorname{tr}_{N-1}\right) \mathbb{E}\left[R_{N-1}(w-\gamma)\right] \\
& \quad=\left(\operatorname{id}_{m} \otimes \phi\right)\left(\left((w-\gamma) \otimes 1_{\mathcal{A}}-\alpha \otimes x-\beta \otimes a_{N-1}\right)^{-1}\right)+O(1 / N) .
\end{aligned}
$$

This result still holds for $w \in M_{m}(\mathbb{C})$ such that $\Im w<0$. 
Proof. By (5.31), it is sufficient to prove that

$$
\begin{aligned}
\left(\operatorname{id}_{m}\right. & \left.\otimes t_{N-1}\right) \mathbb{E}\left[\hat{R}_{N-1}(w-\gamma)\right] \\
& =\left(\operatorname{id}_{m} \otimes \phi\right)\left(\left((w-\gamma) \otimes 1_{\mathcal{A}}-\alpha \otimes x-\beta \otimes a_{N-1}\right)^{-1}\right)+O(1 / N) .
\end{aligned}
$$

According to Theorem 5.7 in [11, we have

$$
\begin{aligned}
& \left(\operatorname{id}_{m} \otimes \operatorname{tr}_{N-1}\right) \mathbb{E}\left[\hat{R}_{N-1}(w-\gamma)\right] \\
& \quad-\left(\operatorname{id}_{\mathrm{m}} \otimes \phi\right)\left(\left((w-\gamma) \otimes 1_{\mathcal{A}}-\alpha \otimes x-\beta \otimes a_{N-1}\right)^{-1}\right)+E_{N-1}(w)=O\left(\frac{1}{N \sqrt{N}}\right),
\end{aligned}
$$

where $E_{N-1}(w)$ is given by

$$
\begin{aligned}
& E_{N-1}(w)= \\
& \tilde{G}_{N-1}^{\prime}(w) \cdot \alpha L_{N-1}(w) \alpha-\frac{1}{2} \tilde{G}_{N-1}^{\prime \prime}(w) \cdot\left(\alpha L_{N-1}(w) \alpha, \alpha L_{N-1}(w) \alpha\right)-L_{N-1}(w)
\end{aligned}
$$

with

$$
L_{N-1}(w)=\frac{1}{N-1} \sum_{j=1}^{N-1}\left(Y_{N-1}(w) \Psi(w)\right)_{j j},
$$

$\Psi, Y_{N-1}$ and $\tilde{G}_{N-1}$ being defined in Theorem 5.3 [11, (5.32) and (5.34) respectively. Set

$$
\begin{aligned}
T_{N} & =\frac{1}{2 \sqrt{2}(N-1)^{2} \sqrt{N-1}} \kappa_{3}(1-\sqrt{-1}) \\
& \times \sum_{i, j, l=1}^{N-1}\left(Y_{N-1}(w)\right)_{j l} \mathbb{E}\left\{\alpha\left(\hat{R}_{N-1}(w)\right)_{i i} \alpha\left(\hat{R}_{N-1}(w)\right)_{l l} \alpha\left(\hat{R}_{N-1}(w)\right)_{i j}\right\},
\end{aligned}
$$

where $\kappa_{3}$ still denotes the third cumulant of $\mu$. Using Cauchy-Schwartz inequality, the bounds (5.33), (5.30) and [11, Lemme 8.1, (8.14)], it can be easily proven that

$$
L_{N-1}(w)-T_{N}=O(1 / N)
$$


Note moreover that, for any $m \times m$ matrix $B$ with bounded operator norm

$$
\begin{aligned}
\operatorname{Tr}_{m}\left(B T_{N}\right)= & \frac{1}{2 \sqrt{2}(N-1)^{2} \sqrt{N-1}} \kappa_{3}(1-\sqrt{-1}) \\
& \times \sum_{i, j, l=1}^{N-1} \operatorname{Tr}_{m} \mathbb{E}\left\{\alpha\left(\hat{R}_{N-1}(w)\right)_{i i} \alpha\left(\hat{R}_{N-1}(w)\right)_{l l} \alpha\left(\hat{R}_{N-1}(w)\right)_{i j}\right. \\
= & \frac{1}{2 \sqrt{2}(N-1)^{2} \sqrt{N-1}} \kappa_{3}(1-\sqrt{-1}) \\
& \times \sum_{i, l=1}^{N-1} \operatorname{Tr}_{m} \mathbb{E}\left\{\alpha\left(\hat{R}_{N-1}(w)\right)_{i i} \alpha\left(\hat{R}_{N-1}(w)\right)_{l l} \alpha\right. \\
& \left.\times\left[\hat{R}_{N-1}(w)\left(B \otimes I_{N-1}\right) Y_{N-1}(w)\right]_{i l}\right\},
\end{aligned}
$$

so that

$$
\begin{aligned}
\left|\operatorname{Tr}_{m}\left(B T_{N}\right)\right| & \\
\leq & \frac{\left|\kappa_{3}\right| m\|\alpha\|^{3}}{2(N-1) \sqrt{N-1}} \\
& \times\left\|(\Im w)^{-1}\right\|^{2} \mathbb{E}\left\{\left(\sum_{i, l=1}^{N-1}\left\|\left[\hat{R}_{N-1}(w)\left(B \otimes I_{N-1}\right) Y_{N-1}(w)\right]_{i l}\right\|^{2}\right)^{1 / 2}\right\} \\
\leq & \frac{\left|\kappa_{3}\right| m\|\alpha\|^{3}\left\|(\Im w)^{-1}\right\|^{4}\|B\|}{2(N-1)} \\
= & O(1 / N),
\end{aligned}
$$

so that

$$
T_{N}=O(1 / N)
$$

and therefore, using (5.5),

$$
E_{N-1}=O(1 / N) .
$$

Lemma 5.5 follows.

\section{Proposition 5.5.}

$$
\begin{aligned}
& \sqrt{N}\left\{\operatorname{id}_{m}\right. \otimes \operatorname{tr}_{N-1} R_{N-1}\left(\rho_{N} e_{11}-\gamma\right) \mathbf{1}_{\tilde{\Omega}_{N-1}} \\
&\left.-\operatorname{id}_{m} \otimes \phi\left(\left(\left(\rho_{N} e_{11}-\gamma\right) \otimes 1_{\mathcal{A}}-\alpha \otimes x-\beta \otimes a_{N-1}\right)^{-1}\right)\right\}
\end{aligned}
$$

goes to zero in probability. 
Proof. Using (4.1), for $N$ large enough, there exists $K>0$ such that

$$
\left\|\left(\rho_{N} e_{11}-\gamma\right) \otimes 1_{\mathcal{A}}-\alpha \otimes x-\beta \otimes a_{N-1}\right\| \leq K
$$

and on $\tilde{\Omega}_{N-1}$,

$$
\left\|\left(\rho_{N} e_{11}-\gamma\right) \otimes I_{N-1}-\alpha \otimes \frac{W_{N-1}}{\sqrt{N}}-\beta \otimes A_{N-1}\right\| \leq K .
$$

Moreover, (see (5.8) and (5.6)), for $N$ large enough,

$$
\left.d\left(0, \operatorname{spect}\left(\rho_{N} e_{11}-\gamma\right) \otimes 1_{\mathcal{A}}-\alpha \otimes x-\beta \otimes a_{N-1}\right)\right)>C_{\epsilon} / 4
$$

and on $\tilde{\Omega}_{N-1}$

$$
d\left(0, \operatorname{spect}\left(\left(\rho_{N} e_{11}-\gamma\right) \otimes I_{N-1}-\alpha \otimes \frac{W_{N-1}}{\sqrt{N}}-\beta \otimes A_{N-1}\right)\right)>C_{\epsilon} / 4 .
$$

Let $g: \mathbb{R} \rightarrow \mathbb{R}$ be a $\mathcal{C}^{\infty}$ function with support in $\left\{C_{\epsilon} / 8 \leq|x| \leq 2 K\right\}$ and such that $g \equiv 1$ on $\left\{C_{\epsilon} / 4 \leq|x| \leq K\right\} . f: x \mapsto \frac{g(x)}{x}$ is a $\mathcal{C}^{\infty}$ function with compact support. Note that

$$
\begin{aligned}
\left(\operatorname{id}_{m} \otimes \phi\right)\left(\left(\left(\rho_{N} e_{11}-\gamma\right) \otimes 1_{\mathcal{A}}-\alpha \otimes x-\beta \otimes a_{N-1}\right)^{-1}\right) \\
\quad=\left(\operatorname{id}_{m} \otimes \phi\right)\left(f\left(\left(\rho_{N} e_{11}-\gamma\right) \otimes 1_{\mathcal{A}}-\alpha \otimes x-\beta \otimes a_{N-1}\right)\right)
\end{aligned}
$$

and on $\tilde{\Omega}_{N-1}$,

$$
R_{N-1}\left(\rho_{N} e_{11}-\gamma\right)=f\left(\left(\rho_{N} e_{11}-\gamma\right) \otimes I_{N-1}-\alpha \otimes \frac{W_{N-1}}{\sqrt{N}}-\beta \otimes A_{N-1}\right) .
$$

According to Lemma 5.5 for any $z \in \mathbb{C} \backslash \mathbb{R}$,

$$
\begin{gathered}
\sqrt{N}\left(\operatorname{id}_{m} \otimes \operatorname{tr}_{N-1}\right) \mathbb{E}\left[R_{N-1}\left(\rho_{N} e_{11}-\gamma-z I_{m}\right)\right] \\
=\sqrt{N}\left(\operatorname{id}_{m} \otimes \phi\right)\left(\left(\left(\rho_{N} e_{11}-\gamma-z I_{m}\right) \otimes 1_{\mathcal{A}}-\alpha \otimes x-\beta \otimes a_{N-1}\right)^{-1}\right)+o^{(z)}(1),
\end{gathered}
$$

where there exist polynomials $Q_{1}$ and $Q_{2}$ with non negative coefficients and $(d, k) \in$ $\mathbb{N}^{2}$ such that

$$
\left\|o^{(z)}(1)\right\| \leq \frac{Q_{1}\left(|\Im z|^{-1}\right)(|z|+1)^{d}}{\sqrt{N}} \leq \frac{1}{\sqrt{N}} \frac{Q_{2}(|\Im z|)(|z|+1)^{d}}{|\Im z|^{k}} .
$$

We recall Helffer-Sjöstrand's representation formula : let $f \in C^{k+1}(\mathbb{R})$ with compact support and $M$ a Hermitian matrix,

$$
f(M)=\frac{1}{\pi} \int_{\mathbb{C}} \bar{\partial} F_{k}(f)(z)(M-z)^{-1} d^{2} z
$$


where $d^{2} z$ denotes the Lebesgue measure on $\mathbb{C}$.

$$
F_{k}(f)(x+i y)=\sum_{l=0}^{k} \frac{(i y)^{l}}{l !} f^{(l)}(x) \chi(y)
$$

where $\chi: \mathbb{R} \rightarrow \mathbb{R}^{+}$is a smooth compactly supported function such that $\chi \equiv 1$ in a neighborhood of 0 , and $\bar{\partial}=\partial_{x}+i \partial_{y}$.

The function $F_{k}(f)$ coincides with $f$ on the real axis and is an extension to the complex plane.

Note that, in a neighborhood of the real axis,

$$
\bar{\partial} F_{k}(f)(x+i y)=\frac{(i y)^{k}}{k !} f^{(k+1)}(x)=O\left(|y|^{k}\right) \text { as } y \rightarrow 0 .
$$

Therefore, by Helffer-Sjöstrand functional calculus,

$$
\begin{gathered}
\sqrt{N}\left(\operatorname{id}_{m} \otimes \operatorname{tr}_{N-1}\right) \mathbb{E}\left(f\left(\left(\rho_{N} e_{11}-\gamma\right) \otimes I_{N-1}-\alpha \otimes \frac{W_{N-1}}{\sqrt{N}}-\beta \otimes A_{N-1}\right)\right) \\
=\frac{1}{\pi} \int_{\mathbb{C} \backslash \mathbb{R}} \bar{\partial} F_{k}(f)(z) \sqrt{N}\left(\operatorname{id}_{m} \otimes t r_{N-1}\right) \mathbb{E}\left[R_{N-1}\left(\rho_{N} e_{11}-\gamma-z I_{m}\right)\right] d^{2} z
\end{gathered}
$$

and

$$
\begin{aligned}
& \sqrt{N}\left(\operatorname{id}_{m} \otimes \phi\right)\left[f\left(\left(\rho_{N} e_{11}-\gamma\right) \otimes I-\alpha \otimes x-\beta \otimes a_{N-1}\right)\right]= \\
& \frac{1}{\pi} \int_{\mathbb{C} \backslash \mathbb{R}} \bar{\partial} F_{k}(f)(z) \sqrt{N}\left(\operatorname{id}_{m} \otimes \phi\right)\left(\left(\left(\rho_{N} e_{11}-\gamma-z I_{m}\right) \otimes 1_{\mathcal{A}}-\alpha \otimes x-\beta \otimes a_{N-1}\right)^{-1}\right) d^{2} z .
\end{aligned}
$$

Hence, using (5.46) and (5.44), we can deduce that

$$
\begin{aligned}
\sqrt{N}\left(\operatorname{id}_{m} \otimes\right. & \left.\operatorname{tr}_{N-1}\right) \mathbb{E}\left(f\left(\left(\rho_{N} e_{11}-\gamma\right) \otimes I_{N-1}-\alpha \otimes \frac{W_{N-1}}{\sqrt{N}}-\beta \otimes A_{N-1}\right)\right) \\
= & \sqrt{N}\left(\operatorname{id}_{m} \otimes \phi\right)\left(\left(\left(\rho_{N} e_{11}-\gamma\right) \otimes I-\alpha \otimes x-\beta \otimes a_{N-1}\right)^{-1}\right) \\
& \quad \frac{1}{\pi} \int_{z \in \mathbb{C} \backslash \mathbb{R}} \partial F_{k}(f)(z) o^{(z)}(1) d^{2} z .
\end{aligned}
$$

Note that since $f$ and $\chi$ are compactly supported, the last integral is an integral on a bounded set of $\mathbb{C}$ and according to (5.47) and (5.50),

$$
\left\|\frac{1}{\pi} \int_{\mathbb{C} \backslash \mathbb{R}} \partial F_{k}(f)(z) o^{(z)}(1) d^{2} z\right\| \leq \frac{C}{\sqrt{N}} .
$$

Thus,

$$
\sqrt{N}\left\{\mathbb{E}\left(\operatorname{id}_{m} \otimes \operatorname{tr}_{N-1}\right)\left(f\left(\left(\rho_{N} e_{11}-\gamma\right) \otimes I_{N-1}-\alpha \otimes \frac{W_{N-1}}{\sqrt{N}}-\beta \otimes A_{N-1}\right)\right)\right.
$$




$$
\left.-\left(\operatorname{id}_{\mathrm{m}} \otimes \phi\right)\left(\left(\left(\rho_{N} e_{11}-\gamma\right) \otimes 1_{\mathcal{A}}-\alpha \otimes x-\beta \otimes a_{N-1}\right)^{-1}\right)\right\} \rightarrow_{N \rightarrow+\infty} 0 .
$$

Now, we are going to study the concentration of

$$
\sqrt{N}\left(\operatorname{id}_{m} \otimes \operatorname{tr}_{N-1}\right)\left(f\left(\left(\rho_{N} e_{11}-\gamma\right) \otimes I_{N-1}-\alpha \otimes \frac{W_{N-1}}{\sqrt{N}}-\beta \otimes A_{N-1}\right)\right)
$$

around its expectation. Define for any $(p, q) \in\{1, \ldots, m\}^{2}, h_{p q}: M_{N-1}^{s a}(\mathbb{C}) \rightarrow \mathbb{C}$ by

$$
\begin{aligned}
& h_{p q}(X) \\
& =\frac{1}{N-1}\left(\operatorname{Tr}_{m} \otimes \operatorname{Tr}_{N-1}\right)\left[\left(e_{q p} \otimes I_{N-1}\right) f\left(\left(\rho_{N} e_{11}-\gamma\right) \otimes I_{N-1}-\alpha \otimes X-\beta \otimes A_{N-1}\right)\right],
\end{aligned}
$$

so that

$$
\left(\mathrm{id}_{m} \otimes \operatorname{tr}_{N-1}\right)\left[f\left(\left(\rho_{N} e_{11}-\gamma\right) \otimes I_{N-1}-\alpha \otimes X-\beta \otimes A_{N-1}\right)\right]=\sum_{p, q=1}^{m} h_{p q} e_{p q} .
$$

Define also $\tilde{h}_{p q}: \mathbb{R}^{(N-1)^{2}} \rightarrow \mathbb{C}$ by $\tilde{h}_{p q}=h_{p q} \circ \Psi^{-1}$, where $\Psi$ is defined in (5.28). Note that

$$
\left\|\nabla \tilde{h}_{p q}(\Psi(X))\right\|=\left\|\operatorname{grad} h_{p q}(X)\right\|_{e} .
$$

Applying Poincaré inequality for $\tilde{h}_{p q}$, we get that

$$
\mathbb{E}\left(\left|h_{p q}\left(\frac{W_{N-1}}{\sqrt{N}}\right)-\mathbb{E}\left(h_{p q}\left(\frac{W_{N-1}}{\sqrt{N}}\right)\right)\right|^{2}\right) \leq \frac{C}{N} \mathbb{E}\left(\left\|\operatorname{grad} h_{p q}\left(\frac{W_{N-1}}{\sqrt{N}}\right)\right\|_{e}^{2}\right),
$$

with

$$
\left\|\operatorname{grad} h_{p q}(X)\right\|_{e}^{2}=\sup _{w \in S_{1}\left(M_{N-1}^{s a}(\mathbb{C})\right)}\left|\frac{d}{d t} h_{p q}(X+t w)_{\mid t=0}\right|^{2} .
$$

For $w$ in $S_{1}\left(M_{N-1}^{s a}(\mathbb{C})\right)$, set

$$
\begin{aligned}
\Delta(t)= & f\left(\left(\rho_{N} e_{11}-\gamma\right) \otimes I_{N-1}-\alpha \otimes(X+t w)-\beta \otimes A_{N-1}\right) \\
& -f\left(\left(\rho_{N} e_{11}-\gamma\right) \otimes I_{N-1}-\alpha \otimes X-\beta \otimes A_{N-1}\right)
\end{aligned}
$$

and

$$
\Delta(t)=\sum_{p^{\prime}, q^{\prime} \in\{1, \ldots, m\}^{2}} e_{p^{\prime} q^{\prime}} \otimes \Delta_{p^{\prime} q^{\prime}}(t) .
$$

Note that $\Delta(t)=\Delta(t)^{*}$ so that $\Delta_{q^{\prime} p^{\prime}}(t)=\Delta_{p^{\prime} q^{\prime}}(t)^{*}$ We have

$$
\left|\frac{d}{d t} h_{p q}(X+t w)_{\mid t=0}\right|^{2}=\left|\lim _{t \rightarrow 0} \frac{1}{t} \operatorname{tr}_{N-1} \Delta_{p q}(t)\right|^{2} .
$$

Moreover, we have

$$
\begin{aligned}
\left(\operatorname{Tr}_{m} \otimes \operatorname{Tr}_{N-1}\right) \Delta(t)^{2} & =\sum_{p, q=1}^{m} \operatorname{Tr}_{N-1} \Delta_{p q}(t) \Delta_{q p}(t) \\
& =\sum_{p, q=1}^{m} \operatorname{Tr}_{N-1} \Delta_{p q}(t) \Delta_{p q}(t)^{*}
\end{aligned}
$$


Therefore $\operatorname{Tr}_{N-1} \Delta_{p q}(t) \Delta_{p q}(t)^{*} \leq\left(\operatorname{Tr}_{m} \otimes \operatorname{Tr}_{N-1}\right) \Delta^{2}(t)$. Since $f$ is a Lipschitz function on $\mathbb{R}$ with Lipschitz constant $C_{L}$, its extension on Hermitian matrices is $C_{L^{-}}$ Lipschitz with respect to the norm $\|M\|_{e}=\left(\operatorname{Tr}_{m(N-1)} M^{2}\right)^{1 / 2}$. Thus,

$$
\begin{aligned}
\left|\operatorname{tr}_{N-1} \Delta_{p q}(t)\right|^{2} & \leq \operatorname{tr}_{N-1} \Delta_{p q}(t) \Delta_{p q}(t)^{*} \\
& \leq \frac{1}{N-1}\left(\operatorname{Tr}_{m} \otimes \operatorname{Tr}_{N-1}\right) \Delta(t)^{2} \\
& \leq C_{L}^{2} \frac{t^{2}}{N-1}\left(\operatorname{Tr}_{m} \otimes \operatorname{Tr}_{N-1}\right)\left(\alpha^{2} \otimes w^{2}\right)=t^{2} \frac{1}{N-1} C_{L}^{2} \operatorname{Tr}_{m} \alpha^{2}
\end{aligned}
$$

Therefore,

$$
\sup _{w \in S_{1}\left(M_{N-1}^{s a}(\mathbb{C})\right)}\left|\frac{d}{d t} h_{p q}(X+t w)_{\mid t=0}\right|^{2} \leq \frac{C}{N},
$$

and then

$$
\mathbb{E}\left(\left|\sqrt{N}\left\{h_{p q}\left(\frac{W_{N-1}}{\sqrt{N}}\right)-\mathbb{E}\left(h_{p q}\left(\frac{W_{N-1}}{\sqrt{N}}\right)\right)\right\}\right|^{2}\right) \leq \frac{C}{N} .
$$

It readily follows that

$$
\begin{gathered}
\sqrt{N}\left(\operatorname{id}_{m} \otimes \operatorname{tr}_{N-1}\right)\left(f\left(\left(\rho_{N} e_{11}-\gamma\right) \otimes I_{N-1}-\alpha \otimes \frac{W_{N-1}}{\sqrt{N}}-\beta \otimes A_{N-1}\right)\right) \\
-\sqrt{N} \mathbb{E}\left(\operatorname{id}_{m} \otimes \operatorname{tr}_{N-1}\right)\left(f\left(\left(\rho_{N} e_{11}-\gamma\right) \otimes I_{N-1}-\alpha \otimes \frac{W_{N-1}}{\sqrt{N}}-\beta \otimes A_{N-1}\right)\right) \\
=o_{\mathbb{P}}(1) .
\end{gathered}
$$

Proposition 5.5 follows from (5.45), (5.51), (5.52) and (5.23).

\section{Proof of Theorem 4.1}

According to Lemma 2.1 $\lambda \in R$ is an eigenvalue of $M_{N}$ if and only if

$$
\operatorname{det}\left(\lambda e_{11} \otimes I_{N}-\gamma \otimes I_{N}-\alpha \otimes \frac{W_{N}}{\sqrt{N}}-\beta \otimes A_{N}\right)=0
$$

or, since there exist permutation matrices $K_{N m}$ and $K_{m N}$ in $M_{N m}$ such that for any $A \in M_{N}$ and $B \in M_{m}$,

$$
A \otimes B=K_{N m}(B \otimes A) K_{m N},
$$

equivalently

$$
\operatorname{det}\left(I_{N} \otimes\left(\lambda e_{11}-\gamma\right)-\frac{W_{N}}{\sqrt{N}} \otimes \alpha-A_{N} \otimes \beta\right)=0 .
$$

Thus, $\lambda$ is an eigenvalue of $M_{N}$ if and only if

$$
\exists V \in \mathbb{C}^{N m} \backslash\{0\},\left(I_{N} \otimes\left(\lambda e_{11}-\gamma\right)-\frac{W_{N}}{\sqrt{N}} \otimes \alpha-A_{N} \otimes \beta\right) V=0 .
$$


Set

$$
V=\sum_{i=1}^{m} V_{i} \otimes e_{i}
$$

where $\left(e_{i}\right)_{i=1, \ldots, m}$ is the canonical basis of $\mathbb{C}^{m}$ and

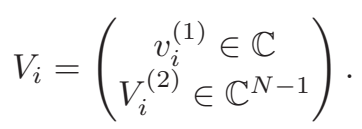

(6.2) can be rewritten

$$
\begin{aligned}
\sum_{i=1}^{m}\left\{\left(\begin{array}{c}
v_{i}^{(1)}\left(\lambda e_{11}-\gamma\right) e_{i} \\
V_{i}^{(2)} \otimes\left(\lambda e_{11}-\gamma\right) e_{i}
\end{array}\right)\right. & -\left(\begin{array}{c}
\left(\frac{W_{11}}{\sqrt{N}} v_{i}^{(1)}+\frac{Y^{*}}{\sqrt{N}} V_{i}^{(2)}\right) \alpha e_{i} \\
\left(\frac{Y}{\sqrt{N}} v_{i}^{(1)}+\frac{W_{N-1}}{\sqrt{N}} V_{i}^{(2)}\right) \otimes \alpha e_{i}
\end{array}\right) \\
& \left.-\left(\begin{array}{c}
\theta v_{i}^{(1)} \beta e_{i} \\
A_{N-1} V_{i}^{(2)} \otimes \beta e_{i}
\end{array}\right)\right\}=0
\end{aligned}
$$

which leads to the system

$$
\left\{\begin{array}{c}
\left(\lambda e_{11}-\gamma-\alpha \frac{W_{11}}{\sqrt{N}}-\beta \theta\right)\left(\sum_{i=1}^{m} v_{i}^{(1)} e_{i}\right)=\left(\frac{Y^{*}}{\sqrt{N}} \otimes \alpha\right)\left(\sum_{i=1}^{m} V_{i}^{(2)} \otimes e_{i}\right) \\
\left(I_{N-1} \otimes\left(\lambda e_{11}-\gamma\right)-\frac{W_{N-1}}{\sqrt{N}} \otimes \alpha-A_{N-1} \otimes \beta\right)\left(\sum_{i=1}^{m} V_{i}^{(2)} \otimes e_{i}\right) \\
=\left(\frac{Y}{\sqrt{N}} \otimes \alpha\right)\left(\sum_{i=1}^{m} v_{i}^{(1)} e_{i}\right)
\end{array}\right.
$$

Let $\tau$ be defined by (4.9). For any $\lambda \in B(\rho, \tau)$, according to Lemma 5.1 and (6.1), we can define on $\tilde{\Omega}_{N-1}$

$$
\tilde{R}_{N-1}\left(\lambda e_{11}-\gamma\right)=\left(I_{N-1} \otimes\left(\lambda e_{11}-\gamma\right)-\frac{W_{N-1}}{\sqrt{N}} \otimes \alpha-A_{N-1} \otimes \beta\right)^{-1} .
$$

The following lines hold on $\Omega_{N}$ (defined by (4.10) ).

First, we can deduce from the above system that $\lambda \in B(\rho, \tau)$ is an eigenvalue of $M_{N}$ if and only if there exists $\left(v_{i}^{(1)}\right)_{i=1, \ldots, m} \in \mathbb{C}^{m},\left(V_{i}^{(2)}\right)_{i=1, \ldots, m} \in \mathbb{C}^{m(N-1)}$, such that:

$$
\begin{gathered}
\sum_{i=1}^{m} v_{i}^{(1)} e_{i} \neq 0 \\
\sum_{i=1}^{m} V_{i}^{(2)} \otimes e_{i}=\tilde{R}_{N-1}\left(\lambda e_{11}-\gamma\right)\left(\frac{Y}{\sqrt{N}} \otimes \alpha\right)\left(\sum_{i=1}^{m} v_{i}^{(1)} e_{i}\right) \\
\left(\lambda e_{11}-\gamma-\alpha \frac{W_{11}}{\sqrt{N}}-\beta \theta-\frac{1}{N}\left(Y^{*} \otimes \alpha\right) \tilde{R}_{N-1}\left(\lambda e_{11}-\gamma\right)(Y \otimes \alpha)\right)\left(\sum_{i=1}^{m} v_{i}^{(1)} e_{i}\right)=0
\end{gathered}
$$

Therefore in particular this implies

$$
\operatorname{det}\left(X_{m}(N)\right)=0
$$


where

$X_{m}(N)=\lambda(N, \rho) e_{11}-\gamma-\alpha \frac{W_{11}}{\sqrt{N}}-\beta \theta-\frac{1}{N}\left(Y^{*} \otimes \alpha\right) \tilde{R}_{N-1}\left(\lambda(N, \rho) e_{11}-\gamma\right)(Y \otimes \alpha)$,

with $\lambda(N, \rho)$ defined by (4.11). Now, noticing that

$$
\begin{gathered}
\left(Y^{*} \otimes \alpha\right) \tilde{R}_{N-1}\left(\lambda(N, \rho) e_{11}-\gamma\right)(Y \otimes \alpha) \\
=\left(\operatorname{Tr}_{N-1} \otimes \operatorname{id}_{m}\right)\left[\left(E_{11} \otimes I_{m}\right)\left(\tilde{Y}^{*} \otimes \alpha\right) \tilde{R}_{N-1}\left(\lambda(N, \rho) e_{11}-\gamma\right)(\tilde{Y} \otimes \alpha)\right],
\end{gathered}
$$

where $\tilde{Y}=(Y \mid 0) \in M_{N-1}(\mathbb{C})$, and using (6.1), it is easy to see that

$$
\begin{aligned}
& \left(Y^{*} \otimes \alpha\right) \tilde{R}_{N-1}\left(\lambda(N, \rho) e_{11}-\gamma\right)(Y \otimes \alpha) \\
& \quad=\left(\alpha \otimes Y^{*}\right) R_{N-1}\left(\lambda(N, \rho) e_{11}-\gamma\right)(\alpha \otimes Y) .
\end{aligned}
$$

Let $\rho_{N}$ be as defined by (4.15). Using the identity

$$
\begin{gathered}
R_{N-1}\left(\rho_{N} e_{11}-\gamma\right)-R_{N-1}\left(\lambda(N, \rho) e_{11}-\gamma\right) \\
=\left(\lambda(N, \rho)-\rho_{N}\right) R_{N-1}\left(\rho_{N} e_{11}-\gamma\right)\left(e_{11} \otimes I_{N-1}\right) R_{N-1}\left(\lambda(N, \rho) e_{11}-\gamma\right),
\end{gathered}
$$

we have

$$
X_{m}(N)=H_{m}(N)+X_{m}^{(0)}(N)
$$

where

$$
X_{m}^{(0)}(N)=\omega_{m}^{(N)}\left(\rho_{N} e_{11}-\gamma\right)-\beta \theta,
$$

$\left(\omega_{m}^{(N)}\right.$ is defined by (4.14) $)$,

$$
\begin{aligned}
H_{m}(N)= & \left(\lambda(N, \rho)-\rho_{N}\right) e_{11}-\Delta_{1}(N)-\Delta_{2}(N) \\
& +\left(\lambda(N, \rho)-\rho_{N}\right) r_{1}(N)-\alpha \frac{W_{11}}{\sqrt{N}}-\left(\lambda(N, \rho)-\rho_{N}\right)^{2} r_{2}(N)
\end{aligned}
$$

with

$$
\begin{aligned}
& r_{1}(N) \\
& =\frac{1}{N}\left(\alpha \otimes Y^{*}\right) R_{N-1}\left(\rho_{N} e_{11}-\gamma\right) \mathbf{1}_{\tilde{\Omega}_{N-1}}\left(e_{11} \otimes I_{N-1}\right) R_{N-1}\left(\rho_{N} e_{11}-\gamma\right) \mathbf{1}_{\tilde{\Omega}_{N-1}}(\alpha \otimes Y), \\
& r_{2}(N)=\frac{1}{N}\left(\alpha \otimes Y^{*}\right) R_{N-1}\left(\rho_{N} e_{11}-\gamma\right) \mathbf{1}_{\tilde{\Omega}_{N-1}}\left(e_{11} \otimes I_{N-1}\right) R_{N-1}\left(\rho_{N} e_{11}-\gamma\right) \mathbf{1}_{\tilde{\Omega}_{N-1}} \\
& \times\left(e_{11} \otimes I_{N-1}\right) R_{N-1}\left(\lambda(N, \rho) e_{11}-\gamma\right) \mathbf{1}_{\tilde{\Omega}_{N-1}}(\alpha \otimes Y), \\
& \Delta_{1}(N)=\frac{1}{N}\left(\alpha \otimes Y^{*}\right) R_{N-1}\left(\rho_{N} e_{11}-\gamma\right) \mathbf{1}_{\tilde{\Omega}_{N-1}}(\alpha \otimes Y) \\
& -\alpha\left(\operatorname{id}_{m} \otimes \operatorname{tr}_{N-1}\right)\left(\left(R_{N-1}\left(\rho_{N} e_{11}-\gamma\right) \mathbf{1}_{\tilde{\Omega}_{N-1}}\right)\right) \alpha,
\end{aligned}
$$




$$
\begin{aligned}
\Delta_{2}(N) & =\alpha\left(\operatorname{id}_{m} \otimes \operatorname{tr}_{N-1}\right)\left(R_{N-1}\left(\rho_{N} e_{11}-\gamma\right) \mathbf{1}_{\tilde{\Omega}_{N-1}}\right) \alpha \\
& -\alpha\left(\operatorname{id}_{m} \otimes \phi\right)\left(\left(\left(\rho_{N} e_{11}-\gamma\right) \otimes 1_{\mathcal{A}}-\alpha \otimes x-\beta \otimes a_{N-1}\right)^{-1}\right) \alpha .
\end{aligned}
$$

First, we have that, according to Lemma 5.4 and using (5.6),

$$
\begin{gathered}
r_{1}(N)-\alpha\left(\operatorname{id}_{m} \otimes \operatorname{tr}_{N-1}\right)\left(R_{N-1}\left(\rho_{N} e_{11}-\gamma\right) \mathbf{1}_{\tilde{\Omega}_{N-1}}\left(e_{11} \otimes I_{N-1}\right) R_{N-1}\left(\rho_{N} e_{11}-\gamma\right) \mathbf{1}_{\tilde{\Omega}_{N-1}}\right) \alpha \\
=o_{\mathbb{P}}(1) .
\end{gathered}
$$

From Lemma 5.2, almost surely,

$$
\begin{gathered}
\left(\operatorname{id}_{m} \otimes \operatorname{tr}_{N-1}\right)\left(R_{N-1}\left(\rho_{N} e_{11}-\gamma\right) \mathbf{1}_{\tilde{\Omega}_{N-1}}\left(e_{11} \otimes I_{N-1}\right) R_{N-1}\left(\rho_{N} e_{11}-\gamma\right) \mathbf{1}_{\tilde{\Omega}_{N-1}}\right) \\
\underset{N \rightarrow \infty}{\longrightarrow}\left(\operatorname{id}_{m} \otimes \phi\right)\left(R_{\infty}\left(\rho e_{11}-\gamma\right)\left(e_{11} \otimes 1_{\mathcal{A}}\right) R_{\infty}\left(\rho e_{11}-\gamma\right)\right) .
\end{gathered}
$$

Therefore,

$$
r_{1}(N) \underset{N \rightarrow \infty}{\stackrel{\mathbb{P}}{\longrightarrow}} \alpha\left(\operatorname{id}_{m} \otimes \phi\right)\left(R_{\infty}\left(\rho e_{11}-\gamma\right)\left(e_{11} \otimes 1_{\mathcal{A}}\right) R_{\infty}\left(\rho e_{11}-\gamma\right)\right) \alpha
$$

Now,

$$
\left\|r_{2}(N)\right\| \leq m^{2}\|\alpha\|^{2}\left\|R_{N-1}\left(\rho_{N} e_{11}-\gamma\right) \mathbf{1}_{\tilde{\Omega}_{N-1}}\right\|^{2}\left\|R_{N}\left(\lambda(N, \rho) e_{11}-\gamma\right) \mathbf{1}_{\tilde{\Omega}_{N-1}}\right\| \frac{\|Y\|^{2}}{N} .
$$

By the law of large numbers,

$$
\frac{\|Y\|^{2}}{N}=\frac{1}{N} \sum_{j=2}^{N}\left|W_{j 1}\right|^{2}=1+o_{\mathbb{P}}(1) .
$$

Moreover, by Lemma 5.1, we have

$$
\left\|R_{N-1}\left(\rho_{N} e_{11}-\gamma\right) \mathbf{1}_{\tilde{\Omega}_{N-1}}\right\| \leq 2 / C_{\epsilon} \text { and }\left\|R_{N-1}\left(\lambda(N, \rho) e_{11}-\gamma\right) \mathbf{1}_{\tilde{\Omega}_{N-1}}\right\| \leq 2 / C_{\epsilon} .
$$

Therefore, there exists $C>0$ such that

$$
\mathbb{P}\left(\left\|r_{2}(N)\right\|>C\right) \rightarrow_{N \rightarrow+\infty} 0 .
$$

By Lemma 5.4.

$$
\Delta_{1}(N)=o_{\mathbb{P}}(1) .
$$

Now, Proposition 5.5 readily yields

$$
\sqrt{N} \Delta_{2}(N)=o_{\mathbb{P}}(1) .
$$

Thus (4.12), (4.16), (6.7), (6.8), (6.9) and (6.10) yield that

$$
H_{m}(N)=o_{\mathbb{P}}(1)
$$


Therefore, according to Lemma A.2 (using (4.16), (5.8) and (6.11)), (6.6) and (4.15), with a probability going to one as $N$ goes to infinity,

$$
\begin{aligned}
0 & =\operatorname{det} X_{m}(N) \\
& =\operatorname{det}\left(X_{m}^{(0)}(N)+H_{m}(N)\right) \\
& =\operatorname{det}\left(X_{m}^{(0)}(N)\right)+\operatorname{Tr}_{m}\left[B_{X_{m}^{(0)}(N)} H_{m}(N)\right]+\epsilon_{N} \\
& =\operatorname{Tr}_{m}\left[B_{X_{m}^{(0)}(N)} H_{m}(N)\right]+\epsilon_{N},
\end{aligned}
$$

where

$$
\begin{gathered}
B_{X_{m}^{(0)}(N)}={ }^{t} \operatorname{com}\left(X_{m}^{(0)}(N)\right), \\
\epsilon_{N}=O\left(\left\|H_{m}(N)\right\|^{2}\right) .
\end{gathered}
$$

Thus, using (4.12), (4.16), (6.7), (6.8), (6.10) and Proposition 5.1,

$$
\sqrt{N} \epsilon_{N}=o_{\mathbb{P}}\left(\sqrt{N}\left(\lambda-\rho_{N}\right)\right)+o_{\mathbb{P}}(1)
$$

Hence, with a probability going to one as $N$ goes to infinity,

$$
\begin{gathered}
\sqrt{N}\left(\lambda(N, \rho)-\rho_{N}\right)\left[T r_{m} B_{X_{m}^{(0)}(N)} e_{11}+\operatorname{Tr}_{m} B_{X_{m}^{(0)}(N)} r_{1}(N)+o_{\mathbb{P}}(1)\right] \\
=T r_{m} B_{X_{m}^{(0)}(N)} \sqrt{N} \Delta_{1}(N)+W_{11} T r_{m} B_{X_{m}^{(0)}(N)} \alpha+o_{\mathbb{P}}(1) .
\end{gathered}
$$

Theorem 4.1 readily follows from Proposition 5.1 the independence of $\Delta_{1}(N)$ and $W_{11}$ and the fact that $\omega_{m}^{(N)}\left(\rho_{N} e_{11}-\gamma\right)$ converges towards $\omega_{m}\left(\rho e_{11}-\gamma\right)$ when $N$ goes to infinity (see 3) Lemma 5.2).

\section{Appendix}

A probability measure $\mu$ satisfies a Poincaré inequality if there exists some constant $C_{P I}>0$ such that for any $\mathcal{C}^{1}$ function $f: \mathbb{R} \rightarrow \mathbb{C}$ such that $f$ and $f^{\prime}$ are in $L^{2}(\mu)$,

$$
\mathbf{V}(f) \leq C_{P I} \int\left|f^{\prime}\right|^{2} d \mu,
$$

with $\mathbf{V}(f)=\int\left|f-\int f d \mu\right|^{2} d \mu$.

If the law of a random variable $X$ satisfies the Poincaré inequality with constant $C_{P I}$ then, for any fixed $\alpha \neq 0$, the law of $\alpha X$ satisfies the Poincaré inequality with constant $\alpha^{2} C_{P I}$.

Assume that probability measures $\mu_{1}, \ldots, \mu_{M}$ on $\mathbb{R}$ satisfy the Poincaré inequality with constant $C_{P I}(1), \ldots, C_{P I}(M)$ respectively. Then the product measure $\mu_{1} \otimes$ $\cdots \otimes \mu_{M}$ on $\mathbb{R}^{M}$ satisfies the Poincaré inequality with constant $C_{P I}^{*}=\max _{i \in\{1, \ldots, M\}} C_{P I}(i)$ in the sense that for any differentiable function $f$ such that $f$ and its gradient $\operatorname{grad} f$ are in $L^{2}\left(\mu_{1} \otimes \cdots \otimes \mu_{M}\right)$,

$$
\mathbf{V}(f) \leq C_{P I}^{*} \int\|\operatorname{grad} f\|^{2} d \mu_{1} \otimes \cdots \otimes \mu_{M}
$$

with $\mathbf{V}(f)=\int\left|f-\int f d \mu_{1} \otimes \cdots \otimes \mu_{M}\right|^{2} d \mu_{1} \otimes \cdots \otimes \mu_{M}$. 
Lemma A.1. Lemma 4.4.3 and Exercise 4.4.5 in [2] or Chapter 3 in [31]. Let $\mathbb{P}$ be a probability measure on $\mathbb{R}^{\mathbb{M}}$ which satisfies a Poincaré inequality with constant $C_{P I}$. Then there exists $K_{1}>0$ and $K_{2}>0$ such that, for any Lipschitz function $F$ on $\mathbb{R}^{M}$ with Lipschitz constant $|F|_{\text {Lip }}$,

$$
\forall \epsilon>0, \mathbb{P}\left(\left|F-\mathbb{E}_{\mathbb{P}}(F)\right|>\epsilon\right) \leq K_{1} \exp \left(-K_{2} \frac{\epsilon}{\sqrt{C_{P I}}|F|_{\text {Lip }}}\right) .
$$

Lemma A.2. Let $A$ and $H$ be $m \times m$ matrices such that, for some $K>0$,

$$
\|A\| \leq K,\|H\| \leq K
$$

Then

$$
\operatorname{det}(A+H)=\operatorname{det}(A)+\operatorname{Tr}_{m}\left({ }^{t} \operatorname{com}(A) H\right)+\epsilon
$$

where $\operatorname{com}(A)$ denotes the comatrix of $A$ and there exists a constant $C_{m, K}>0$, only depending on $m$ and $K$, such that $|\epsilon| \leq C_{m, K}\|H\|^{2}$.

Proof. Denote by $a_{1}, \ldots, a_{m}$, resp. $h_{1}, \ldots, h_{m}$, the columns of the matrix $A$, resp. $H$. Since the determinant of a $m \times m$ matrix is a m-linear function of the $m$ columns, we have

$$
\operatorname{det}(A+H)=\operatorname{det}(A)+\sum_{k=1}^{m} \operatorname{det}\left(a_{1}, \ldots, a_{k-1}, h_{k}, a_{k+1}, \ldots, a_{m}\right)+\epsilon,
$$

where $\epsilon$ is the sum of a number only depending on $m$ of determinants involving at least two columns of $H$. Hadamard's inequality and (6.12) readily yields that there exists $C_{m, K}>0$ such that $|\epsilon| \leq C_{m, K}\|H\|^{2}$. Moreover, denoting by $\left\{e_{1}, \ldots, e_{m}\right\}$ the canonical basis of $\mathbb{C}^{m}$, we have

$$
\begin{aligned}
\operatorname{det}\left(a_{1}, \ldots, a_{k-1}, h_{k}, a_{k+1}, \ldots, a_{m}\right) & =\sum_{i=1}^{m} \operatorname{det}\left(a_{1}, \ldots, a_{k-1}, H_{i k} e_{i}, a_{k+1}, \ldots, a_{m}\right) \\
& =\sum_{i=1}^{m} H_{i k}(\operatorname{com} A)_{i k} \\
& \left.={ }^{t}(\operatorname{com} A) H\right)_{k k} .
\end{aligned}
$$

The result readily follows.

\section{Acknowledgments}

I am grateful to Serban Belinschi and Catherine Donati-Martin for useful discussions.

\section{References}

[1] G. W. Anderson. Convergence of the largest singular value of a polynomial in independent Wigner matrices, Ann. Probab. 41(3B) (2013) 2103-2181.

[2] G.W. Anderson, A. Guionnet and O. Zeitouni An introduction to random matrices, Cambridge University Press, Cambridge, 2010. 
[3] M. Anshelevich, S.T. Belinschi, M Février, and A. Nica, Convolution powers in the operator-valued framework, Trans. Amer. Math. Soc. 365, no. 4 (2013) 2063-2097.

[4] Bai, Z. D. and Silverstein, J. W. No eigenvalues outside the support of the limiting spectral distribution of large-dimensional sample covariance matrices, Ann. Probab. 26 (1998) 316-345.

[5] Z. Bai and J. Silverstein, Spectral analysis of large dimensional random matrices (Second edition. Springer Series in Statistics. Springer, New York, 2010).

[6] Z. Bai and J. Yao, Central limit theorem for eigenvalues in a spiked population model, Ann. Inst. H. Poincaré 44 (2008) 447-474.

[7] J. Baik, G. Ben Arous and S. Péché, Phase transition of the largest eigenvalue for nonnull complex sample covariance matrices, Ann. Probab. 33 no. 5 (2005) 16431697.

[8] D. Bakry, I. Gentil, M. Ledoux, Analysis and geometry of Markov diffusion operators. (Grundlehren der Mathematischen Wissenschaften [Fundamental Principles of Mathematical Sciences], 348. Springer, Cham, 2014).

[9] S. T. Belinschi and H. Bercovici, A new approach to subordination results in free probability, J. Anal. Math. 101 (2007) 357-365.

[10] S.T. Belinschi, H. Bercovici, M. Capitaine, M. Février, Outliers in the spectrum of large deformed unitarily invariant models, Ann. Probab. Volume 45 Number 6A (2017) 3571-3625.

[11] S. Belinschi and M. Capitaine, Spectral properties of polynomials in independent Wigner and deterministic. J. Funct. Anal. Volume 273 Issue 12 (2017) 3901-3963.

[12] S. Belinschi, H. Bercovici and M. Capitaine, On the outlying eigenvalues of a polynomial in large independent random matrices. Int. Math. Res. Notices. https://doi.org/10.1093/imrn/rnz080 (2019).

[13] S. Belinschi, T. Mai, R. Speicher, Analytic subordination theory of operator-valued free additive convolution and the solution of a general random matrix problem. J. Reine angew. Math., ISSN (Online) 1435-5345, ISSN (Print) 0075-4102, DOI: 10.1515/crelle-2014-0138, 2015.

[14] H. Bercovici and D. Voiculescu, Free convolution of measures with unbounded support, Indiana Univ. Math. J.42 (1993) 733-773.

[15] P. Biane, Processes with free increments, Math. Z.227 (1998) 143-174, 1998.

[16] M. Capitaine, Additive/multiplicative free subordination property and limiting eigenvectors of spiked additive deformations of Wigner matrices and spiked sample covariance matrices Journal of Theoretical Probability Volume 26 (3) (2013) 595-648.

[17] M. Capitaine, Exact separation phenomenon for the eigenvalues of large InformationPlus-Noise type matrices. Application to spiked models, Indiana Univ. Math. J. 63 (6) (2014) 1875-1910.

[18] M. Capitaine, Limiting eigenvectors of outliers for Spiked Information-Plus-Noise type matrices, Séminaire de Probabilités XLIX Lecture Notes in Mathematics 2215, Springer 2018. 
[19] M. Capitaine, C. Donati-Martin, Spectrum of deformed random matrices and free probability, Advanced Topics in Random Matrices, Florent Benaych-Georges, Charles Bordenave, Mireille Capitaine, Catherine Donati-Martin, Antti Knowles (edited by F. Benaych-Georges, D. Chafai, S. Péché, B. de Tilière, volume Panoramas et Synthèses $53,2018)$.

[20] M. Capitaine, C. Donati-Martin and D. Féral, The largest eigenvalues of finite rank deformation of large Wigner matrices: convergence and nonuniversality of the fluctuations, Ann. Probab. 37, no. 1 (2009) 1-47.

[21] M. Capitaine, C. Donati-Martin and D. Féral, Central limit theorems for eigenvalues of deformations of Wigner matrices, Ann. Inst. H. Poincar Probab. Statist. 48, 1 (2012) 107-133.

[22] M. Capitaine, C. Donati-Martin, D. Féral and M. Février, Free convolution with a semicircular distribution and eigenvalues of spiked deformations of Wigner matrices. Electron. J. Probab. 16 no. 64 (2011) 1750-1792.

[23] M. Capitaine, S. Péché, Fluctuations at the edges of the spectrum of the full rank deformed G.U.E., Probab. Theory Relat. Fields 165(1) (2016) 117-161.

[24] G. P. Chistyakov and F. Götze, Limit theorems in free probability theory. I., Ann. Probab., 36(1) (2008) 54-90.

[25] B. Collins and C. Male, The strong asymptotic freeness of Haar and deterministic matrices, Ann. Sci. Éc. Norm. Supér. (4) 47 no. 1 (2014) 147-163.

[26] P. Deift, Orthogonal polynomials and random matrices: a Riemann-Hilbert approach (volume 3 of Courant Lecture Notes in Mathematics. New York University, Courant Institute of Mathematical Sciences, New York; American Mathematical Society, Providence, RI, 1999).

[27] D. Féral and S. Péché, The largest eigenvalue of rank one deformation of large Wigner matrices, Comm. Math. Phys. 272 no. 1 (2007) 185-228.

[28] P.J Forrester, Log-Gases and Random Matrices (London Mathematical Society Monographs (LMS-34)).

[29] U. Haagerup and S. Thorbjørnsen, A new application of random matrices: $\operatorname{Ext}\left(C_{\text {red }}^{*}\left(\mathbb{F}_{2}\right)\right)$ is not a group, Ann. of Math. (2), 162(2) (2005) 711-775.

[30] U. Haagerup, H. Schultz, and S. Thorbjørnsen, A random matrix approach to the lack of projections in $C_{\text {red }}^{*}\left(\mathbb{F}_{2}\right)$, Adv. Math. 204(1) (2006) 183.

[31] M. Ledoux, The Concentration of Measure Phenomenon. (Mathematical Surveys and Monographs, Volume 89, A.M.S, 2001).

[32] C. Male, The norm of polynomials in large random and deterministic matrices, With an appendix by Dimitri Shlyakhtenko. Probab. Theory Relat. Fields 154 no. 3-4 (2012) 477532 .

[33] H. Maassen, Addition of freely independent random variables, J. Funct. Anal. 106(2) (1992) 409-438.

[34] M.L. Mehta. Random Matrices and the Statistical Theory of Energy Levels (Academic Press, New York, NY, 1967). 
[35] J.A. Mingo and R. Speicher, Free probability and Random matrices (Fields Institute Monographs Originally published by American Mathematical Society ISSN: 10695273. Springer).

[36] A. Nica, D. Shlyakhtenko, and R. Speicher, Operator-Valued Distributions. I. Characterizations of Freeness. Internat. Math. Res. Notices 29 (2002) 1509-1538.

[37] L. Pastur and and M. Shcherbina, Eigenvalue Distribution of Large Random Matrices ( Mathematical Surveys and Monographs, Volume 171, American Mathematical Society).

[38] S. Péché, The largest eigenvalue of small rank perturbations of Hermitian random matrices. Probab. Theory Relat. Fields 134 no. 1 (2006) 127-173.

[39] A. Pizzo, D. Renfrew, A. Soshnikov, On finite rank deformations of Wigner matrices. Ann. Inst. Henri Poincaré Probab. Stat. 49 no. 1 (2013) 64-94.

[40] D. Renfrew and A. Soshnikov, On finite rank deformations of Wigner matrices II. Delocalized perturbations. Random Matrices Theory Appl. 2 no. 11250015 (2013) 36 pp.

[41] R. Speicher, Combinatorial theory of the free product with amalgamation and operatorvalued free probability theory (Mem. Amer. Math. Soc., vol. 132 no. 627, pp. x+88, 1998).

[42] D. Voiculescu. Addition of certain noncommuting random variables, J. Funct. Anal. 66 (1986) 323-346.

[43] D.V. Voiculescu, Limit laws for random matrices and free products, Invent. Math. 104 (1991) 201-220.

[44] D. Voiculescu, The analogues of entropy and of Fisher's information measure in free probability theory. I, Comm. Math. Phys. 155 (1993) 71-92.

[45] D. Voiculescu, Operations on certain noncommutative operator-valued random variables. Recent advances in operator algebras (Orléans, 1992), Astérisque no. 232 (1995) 243-275.

[46] D. Voiculescu.,The coalgebra of the free difference quotient and free probability, Internat. Math. Res. Notices no. 2 (2000) 79-106.

[47] D.V. Voiculescu, K. Dykema, and A. Nica, Free random variables (CRM Monograph Series, vol. 1, American Mathematical Society, Providence, RI, 1992, ISBN 0-82186999-X, A noncommutative probability approach to free products with applications to random matrices, operator algebras and harmonic analysis on free groups). 